\title{
A Note on the Complexity of the Satisfiability Problem for Graded Modal Logics
}

\author{
Yevgeny Kazakov \\ Computing Laboratory \\ Oxford University \\ Parks Rd., Oxford OX1 3QD, England \\ e-mail: yevgeny.kazakov@comlab.ox.ac.uk
}

\author{
Ian Pratt-Hartmann \\ School of Computer Science \\ University of Manchester \\ Oxford Rd., Manchester M13 9PL, England \\ e-mail: ipratt@cs.man.ac.uk
}

\begin{abstract}
Graded modal logic is the formal language obtained from ordinary modal logic by endowing its modal operators with cardinality constraints. Under the familiar possibleworlds semantics, these augmented modal operators receive interpretations such as "It is true at no fewer than 15 accessible worlds that ...", or "It is true at no more than 2 accessible worlds that ...". We investigate the complexity of satisfiability for this language over some familiar classes of frames. This problem is more challenging than its ordinary modal logic counterpart-especially in the case of transitive frames, where graded modal logic lacks the tree-model property. We obtain tight complexity bounds for the problem of determining the satisfiability of a given graded modal logic formula over the classes of frames characterized by any combination of reflexivity, seriality, symmetry, transitivity and the Euclidean property.
\end{abstract}

Keywords-modal logic; graded modalities; computational complexity

\section{INTRODUCTION}

Graded modal logic is the formal language obtained by decorating the $\diamond$-operator of ordinary modal logic with subscripts expressing cardinality constraints. Specifically, for $C \geq 0$, the formula $\nabla_{\leq C} \varphi$ may be glossed: " $\varphi$ is true at no more than $C$ accessible worlds," and the formula $\diamond_{\geq C} \varphi$ may be glossed: " $\varphi$ is true at no fewer than $C$ accessible worlds." The semantics for graded modal logic generalize the relational semantics for ordinary modal logic in the expected way. We employ the labels Rfl, Ser, Sym, Tr and Eucl to denote, respectively, the classes of reflexive, serial, symmetric, transitive and Euclidean frames. (Definitions of these frame classes are given in Table [) Using this notation, $\bigcap\{\mathrm{Rfl}, \mathrm{Tr}\}$ denotes the class of reflexive, transitive frames, $\bigcap\{$ Ser, Tr, Eucl $\}$ denotes the class of serial, transitive, Euclidean frames, and so on. As a limiting case, $\bigcap \emptyset$ denotes the class of all frames. In this paper, we investigate the computational complexity of determining the satisfiability of a given formula of graded modal logic over any frame class of the form $\bigcap \mathcal{F}$, where $\mathcal{F} \subseteq\{$ Rfl, Ser, Sym, Tr, Eucl $\}$.

It is easy to see that ordinary modal logic is in effect a sub-language of graded modal logic: any formula of the form $\diamond \varphi$ may be equivalently written $\diamond_{\geq 1} \varphi$, and similarly, any formula of the form $\square \varphi$ may be equivalently written $\diamond_{\leq 0} \neg \varphi$. And ordinary modal logic provides a good starting point for our analysis, because its complexity-theoretic treatment is comparatively straightforward. The following two theorems are well-known, and may be proved using techniques found in any modern text on modal logic (e.g. [1]). We remind the reader that symmetry and transitivity together imply the Euclidean property.

Theorem 1. Let $\mathcal{F} \subseteq\{$ Rfl, Ser, Sym, Tr, Eucl $\}$, with Eucl $\in$ $\mathcal{F}$ or $\{$ Sym, $\operatorname{Tr}\} \subseteq \mathcal{F}$. Then the satisfiability problem for ordinary modal logic over $\cap \mathcal{F}$ is NP-complete.

Theorem 2. If $\mathcal{F} \subseteq\{\mathrm{Rfl}, \mathrm{Ser}, \mathrm{Tr}\}$, then the satisfiability problem for ordinary modal logic over $\cap \mathcal{F}$ is PSpacecomplete [2]. Also, if $\mathcal{F} \subseteq\{\mathrm{Rfl}, \mathrm{Ser}, \mathrm{Sym}\}$, then the satisfiability problem for ordinary modal logic over $\cap \mathcal{F}$ is PSpace-complete.

The upper complexity bound in Theorem 1 follows from the fact that ordinary modal logic has the polynomial-size model property over the relevant frame classes: if a formula $\varphi$ of ordinary modal logic is satisfiable over a frame in $\bigcap \mathcal{F}$, where $\mathcal{F}$ satisfies the conditions of Theorem 1 then it is satisfiable over a frame in $\bigcap \mathcal{F}$ whose size is bounded by a polynomial function of the number of symbols in $\varphi$. For the frame classes of Theorem 2, ordinary modal logic lacks the polynomial-size model property. However, it does have the tree-model property: if a formula is satisfiable over a frame in any of the classes $\bigcap \mathcal{F}$ mentioned in Theorem 2 then it is satisfiable over a frame in that class which forms a (possibly infinite) tree [3]. Because the branches of this tree can be assumed to be either short or periodic with small period, and because these branches can be explored one-byone, the PSpace-upper complexity bound may be obtained by exhibiting, for each relevant frame class $\bigcap \mathcal{F}$, a suitable semantic tableau algorithm.

Turning our attention to the language of graded modal logic, our first question is whether the results of Theorems 1 and 2 carry over to the larger language. When $\mathcal{F}$ contains neither of the classes Tr or Eucl, the answer is yes. We have:

Theorem 3. The satisfiability problem for graded modal logic over $\mathcal{F}=\bigcap \emptyset$ is PSpace-complete [4]. In fact, if $\mathcal{F} \subseteq$ $\{\mathrm{Rfl}, \mathrm{Ser}, \mathrm{Sym}\}$, then the satisfiability problem for graded modal logic over $\cap \mathcal{F}$ is PSpace-complete. 
The reason-and indeed the reasoning - is essentially the same as for Theorem 22, the PSpace upper complexity bound in Theorem 3 depends on the fact that graded modal logic enjoys the tree-model property over the relevant frame classes. This can then be used to establish the correctness of semantic tableau algorithms for graded modal logic over these frame classes. The paper [4] actually considers only the case $\mathcal{F}=\emptyset$ (i.e. the class of all frames); however, the modifications required to take account of reflexivity, seriality and symmetry are routine, because these restrictions do not compromise the tree-model property. Note that the upper complexity bound in Theorem 3 holds even when numerical subscripts are coded in binary. (The much easier result for unary coding can be found in [5].)

When $\mathcal{F}$ contains either Eucl or $\mathrm{Tr}$, the complexity of the satisfiability problem for graded modal logic over $\bigcap \mathcal{F}$ is harder to determine. Consider first the analogue of Theorem 11. where we have either Eucl $\in \mathcal{F}$ or $\{\operatorname{Tr}, \operatorname{Sym}\} \subseteq \mathcal{F}$, and let $\left\{\varphi_{n}\right\}_{n \geq 0}$ be the sequence of formulas given by $\varphi_{n}=\diamond_{\geq 2^{n}} p$. Assuming binary coding of numerical subscripts, the number of symbols in $\varphi_{n}$ is bounded by a linear function of $n$, and every $\varphi_{n}$ is satisfiable over a Euclidean frame; but $\varphi_{n}$ is certainly not satisfiable over any frame with fewer than $2^{n}$ worlds! Thus, for graded modal logic, the reasoning used to prove Theorem 1 fails. Nevertheless, the corresponding complexity result still holds:

Theorem 4. Let $\mathcal{F} \subseteq\{$ Rfl, Ser, Sym, Tr, Eucl $\}$, with Eucl $\in$ $\mathcal{F}$ or $\{\mathrm{Sym}, \operatorname{Tr}\} \subseteq \mathcal{F}$. Then the satisfiability problem for graded modal logic over $\bigcap \mathcal{F}$ is NP-complete.

We prove Theorem 4 in Section III

When $\mathcal{F}$ contains Tr, but neither Sym nor Eucl, we cannot apply the reasoning of Theorem 2 at all, since graded modal logic lacks the tree-model property over transitive frames. For example, consider the formula $\varphi$ given by

$$
\varphi:=q_{0} \wedge \diamond_{\geq 2}\left(\neg q_{0} \wedge q_{1} \wedge \diamond_{\geq 1}\left(\neg q_{0} \wedge \neg q_{1}\right)\right) \wedge \diamond_{\leq 1} \neg q_{1} .
$$

The formula $\varphi$ is certainly satisfiable over transitive frames; however, it is not satisfiable over tree-shaped transitive frames. For suppose $\varphi$ is true at a world $w_{0}$ in some structure. The conjunct $\diamond_{\geq 2}\left(\neg q_{0} \wedge q_{1} \wedge \diamond_{\geq 1}\left(\neg q_{0} \wedge \neg q_{1}\right)\right)$ ensures the existence of distinct worlds $w_{1}$ and $w_{2}$, accessible from (and distinct from) $w_{0}$, and, for $i=1,2$, a world $w_{i}^{\prime}$ accessible from $w_{i}$ and satisfying $\neg q_{1}$, with $w_{i}^{\prime}$ distinct from $w_{0}, w_{1}$ and $w_{2}$. But the conjunct $\diamond_{\leq 1} \neg q_{1}$ ensures that, if the accessibility relation is transitive, $w_{1}^{\prime}=w_{2}^{\prime}$. Hence, $\varphi$ is not satisfiable over a tree. Indeed, we show below that, for the relevant frame classes, graded modal logic and ordinary modal logic exhibit different complexities:

Theorem 5. Let $\mathcal{F} \subseteq\{\mathrm{Rfl}, \mathrm{Ser}, \operatorname{Tr}\}$, with $\operatorname{Tr} \in \mathcal{F}$. Then the satisfiability problem for graded modal logic over $\cap \mathcal{F}$ is NExpTime-complete. It remains NExpTime-hard, even when all numerical subscripts in modal operators are at most 1.
We prove Theorem 5 in Section IV. The final statement of the theorem is significant, because it means that the result does not depend upon the coding of numerical subscripts.

A moment's thought shows that the conditions in Theorems 3-5] are exhaustive: together, they establish the complexity of the satisfiability problem for graded modal logic over $\bigcap \mathcal{F}$ for every $\mathcal{F} \subseteq\{$ Rfl, Ser, Sym, Tr, Eucl $\}$.

The decidability of the satisfiability problem for graded modal logic over various frame classes $\bigcap \mathcal{F}$ is touched on in [6], where it is stated (p. 520) that "standard techniques or modifications of them may be used to prove the decidability of most of [these] logics"; however, the paper gives no further details. Several such decidability results are claimed in [7]; however, in the (difficult) case where $\mathcal{F}=\{\operatorname{Tr}\}$, this proof contains an error, as reported in [8]. The latter provides a correct proof; however, the method employed there does not establish any complexity bounds. It is conjectured in [9] (Remark 4.12), that the satisfiability problem for graded modal logic over the class of transitive, symmetric and reflexive frames is PSpace-complete: Theorem 4 shows that this conjecture, if true, would imply that PSpace=NP. Earlier accounts of graded modal logics focused primarily on the problem of axiomatizing the set of valid formulas over these frame classes. For instance, [6] provides (or reports) such axiomatizations for $\bigcap \mathcal{F}$, where $\mathcal{F}$ is any of $\emptyset,\{\mathrm{Rfl}\}$, $\{\operatorname{Sym}\},\{$ Rfl, Sym $\},\{R f l, T r\}$ and $\{$ Rfl, Tr, Sym $\}$. Similar results can be found in [10], [11], [12], [13]; see also [9] for axiomatizations of some related logics.

Graded modal logics are closely related to terminological languages and description logics (DLs) [14] featuring socalled qualified number restrictions. These logics allow concepts to be defined by specifying how many things (of various kinds) instances of those concepts can be related to. Logics featuring both qualified number restrictions and transitive relations are frequently undecidable [15], and many DLs incorporate various syntactic restrictions to restore decidability. It was recently shown in [8] that some of these syntactic restrictions can be considerably relaxed.

This paper is an extended version of [16] containing the omitted proofs.

\section{PRELiminaries}

Fix a countably infinite set $\Pi$. The language of graded modal logic is defined to be the smallest set of expressions, $\mathcal{G M}$, satisfying the following conditions:

1) $\Pi \subseteq \mathcal{G M}$;

2) if $\varphi$ and $\psi$ are in $\mathcal{G M}$, then so are $\neg \varphi, \varphi \wedge \psi, \varphi \vee \psi$, $\varphi \rightarrow \psi$ and $\varphi \leftrightarrow \psi$

3) if $\varphi$ is in $\mathcal{G M}$, then so are $\diamond_{\leq C} \varphi$ and $\diamond_{\geq C} \varphi$, for any bit-string $C$.

We refer to expressions in this set as $\mathcal{G} \mathcal{M}$-formulas (or simply formulas, if clear from context). If $\varphi$ is a $\mathcal{G M}$ formula, we take the size of $\varphi$, denoted $\|\varphi\|$, to be the number of symbols in $\varphi$. Throughout the paper, we equivocate 
between bit-strings and the natural numbers they represent in the usual way. Thus, we may informally think of the subscripts in $\nabla_{\leq C}$ and $\diamond_{\geq C}$ as natural numbers, it being understood that the number of symbols in, for example, $\diamond_{\leq C}$ is approximately $\log C$, rather than $C$. That is: in giving the size of a formula, we assume binary, rather than unary, coding.

Let $\Sigma$ be the relational signature with unary predicates $\Pi$ and single binary predicate $r$, and let $\mathfrak{A}$ be a $\Sigma$-structure with domain $W$. We refer to the elements of $W$ as worlds. We define the satisfaction relation for $\mathcal{G} \mathcal{M}$-formulas inductively as follows:

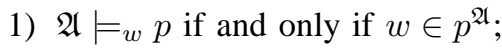

2) $\mathfrak{A} \models_{w} \neg \varphi$ if and only if $\mathfrak{A} \not \models_{w} \varphi$, and similarly for $\wedge, \vee, \rightarrow, \leftrightarrow$

3) $\mathfrak{A} \models_{w} \diamond_{\geq C} \varphi$ if and only if there exist at least $C$ worlds $v \in W$ such that $\langle w, v\rangle \in r^{\mathfrak{A}}$ and $\mathfrak{A} \models{ }_{v} \varphi$;

4) $\mathfrak{A} \models_{w} \diamond_{\leq C} \varphi$ if and only if there exist at most $C$ worlds $v \in W$ such that $\langle w, v\rangle \in r^{\mathfrak{A}}$ and $\mathfrak{A} \models{ }_{v} \varphi$.

The notion of satisfaction extends to sets of $\mathcal{G} \mathcal{M}$-formulas $\Phi$ as expected: $\mathfrak{A} \models_{w} \Phi$ if $\mathfrak{A} \models_{w} \varphi$ for all $\varphi \in \Phi$. If $\mathfrak{A} \models_{w} \varphi$, we sometimes say, informally, that $\varphi$ is true at $w$ in $\mathfrak{A}$. We write $\square \varphi$ as an abbreviation for $\diamond_{\leq 0} \neg \varphi$, and $\diamond_{\varphi}$ as an abbreviation for $\diamond_{\geq 1} \varphi$, or, equivalently, $\neg \diamond_{\leq 0} \varphi$. Thus, the language of ordinary modal logic may be regarded as the subset of $\mathcal{G M}$ in which all indices are restricted to 0 . Finally, we write $\square \varphi$ as an abbreviation for $\varphi \wedge \square \varphi$.

By a frame, we mean an $\{r\}$-structure-in other words, a non-empty (possibly infinite) digraph. If $\mathfrak{A}$ is a $\Sigma$-structure, then its $\{r\}$-reduct is a frame $\mathfrak{F}$ : we say that $\mathfrak{A}$ is a structure over $\mathfrak{F}$. Further, we call the mapping $V: \Pi \rightarrow \mathbb{P}(W)$ given by $p \mapsto p^{\mathfrak{A}}$ the valuation of $\mathfrak{A}$ (on $W$ ). We write $\mathfrak{A}=(W, R, V)$ to indicate that $\mathfrak{A}$ is a $\Sigma$-structure over the frame $(W, R)$ with valuation $V$. Obviously, this determines $\mathfrak{A}$ completely. Henceforth, the term "structure", with no signature qualification, will always mean " $\Sigma$-structure". Let $\varphi$ be a $\mathcal{G M}$-formula. We say that $\varphi$ is satisfiable over a frame $\mathfrak{F}$ if there exists a structure $\mathfrak{A}$ over $\mathfrak{F}$ and a world $w$ of $\mathfrak{A}$ such that $\mathfrak{A} \models_{w} \varphi$. Further, $\varphi$ is satisfiable over a class of frames $\mathcal{K}$ if it is satisfiable over some frame in $\mathcal{K}$. We denote by $\mathcal{G} \mathcal{M}_{\mathcal{K}}$-Sat the problem of determining whether a given $\mathcal{G} \mathcal{M}$-formula is satisfiable over $\mathcal{K}$.

Any first-order sentence $\alpha$ over the signature $\{r\}$ defines a class of frames $\{\mathfrak{F}: \mathfrak{F} \models \alpha\}$. The most common frame classes are those which we agreed in Section I to denote by the labels Rfl, Ser, Sym, Tr and Eucl. Table I] lists these frame classes together with their respective defining first-order sentences. A structure over a reflexive frame will simply be called a reflexive structure, and similarly for the other frame properties. We can now articulate the objective of this paper. Let $\mathcal{F}$ be a subset (possibly empty) of the set of frame classes $\{\mathrm{Rfl}, \mathrm{Ser}, \mathrm{Sym}, \mathrm{Tr}, \mathrm{Eucl}\}$. We ask: what is the complexity of $\mathcal{G} \mathcal{M}_{\cap \mathcal{F}}$-Sat?
Table I: Frame classes considered in this paper.

$$
\begin{array}{ll}
\text { reflexive frames } & \forall x \cdot r(x, x) \\
\text { serial frames } & \forall x \exists y \cdot r(x, y) \\
\text { symmetric frames } & \forall x \forall y \cdot(r(x, y) \rightarrow r(y, x)) \\
\text { transitive frames } & \forall x \forall y \forall z \cdot(r(x, y) \wedge r(y, z) \rightarrow r(x, z)) \\
\text { Euclidean frames } & \forall x \forall y \forall z \cdot(r(x, y) \wedge r(x, z) \rightarrow r(y, z)) \text {. }
\end{array}
$$

\section{EUCLIDEAN FRAMES}

The purpose of this section is to prove Theorem 4. We make use of a known complexity result on first-order logic with counting quantifiers. Denote by $\mathcal{C}^{1}$ the set of firstorder formulas featuring only a single variable $x$, but with the counting quantifiers $\exists_{\leq C} x$ and $\exists_{\geq C} x$ allowed. The following result holds for both unary and binary coding of numerical subscripts:

Theorem 6 ([17], [18]). The problem of deciding satisfiability for $\mathcal{C}^{1}$-formulas is NP-complete.

We show that, for $\mathcal{G M}$-formulas, satisfiability over Euclidean frames is equivalent to satisfiability over frames having a particularly simple form, and that, for such frames, the fragment $\mathcal{C}^{1}$ is as expressive as we need.

Let $\mathfrak{F}=(W, R)$ be a frame. If $X \subseteq W, R(X)$ denotes $\bigcup_{x \in X}\{w \in W \mid\langle x, w\rangle \in R\}$; we write $R(w)$ for $R(\{w\})$. If $\mathfrak{F}=(W, R)$ is a frame, and $X \subseteq W, R^{*}(X)$ denotes $X \cup R(X) \cup R(R(X)) \cup \cdots$; we write $R^{*}(w)$ for $R^{*}(\{w\})$. If $\mathfrak{A}$ is a structure over a frame $(W, R)$ and $X \subseteq W$, let $\mathfrak{B}$ be the substructure of $\mathfrak{A}$ with domain $R^{*}(X)$. We call $\mathfrak{B}$ the substructure generated by $X$. Note that reflexivity, seriality, symmetry, transitivity and the Euclidean property are all preserved under generated substructures.

Lemma 1. Let $\varphi$ be a formula of $\mathcal{G M}, \mathfrak{A}$ a structure, $w$ a world of $\mathfrak{A}$ and $\mathfrak{B}$ the substructure generated by $\{w\}$. If $\mathfrak{A} \models{ }_{w} \varphi$, then $\mathfrak{B} \models{ }_{w} \varphi$.

Proof: Induction on the structure of $\varphi$.

Lemma 2. Let $\mathfrak{F}=(W, R)$ be a Euclidean frame and $w_{0} \in$ $W$. Then: (i) $R\left(w_{0}\right) \subseteq R\left(R\left(w_{0}\right)\right)$, (ii) $R^{*}\left(w_{0}\right)=\left\{w_{0}\right\} \cup$ $R\left(R\left(w_{0}\right)\right)$, and (iii) $R$ is total on $R\left(R\left(w_{0}\right)\right)$.

Proof: For the first statement, observe that, in a Euclidean frame, $R$ is total on any set $R\left(w_{0}\right)$. In particular, $\langle w, w\rangle \in R$ for all $w \in R\left(w_{0}\right)$, whence $R\left(w_{0}\right) \subseteq$ $R\left(R\left(w_{0}\right)\right)$.

Now consider any $X \subseteq W$ such that $R$ is total on $X$. We claim that $R$ is also total on $R(X)$, and that $R(X)=R(R(X))$. By the Euclidean property, $\langle w, w\rangle \in R$ for all $w \in R(X)$, so that $R(X) \subseteq R(R(X))$. We show that $R$ is total on $R(X)$. If $w \in R(X)$ and $R$ is total on $X$, then by the Euclidean property, $\langle x, w\rangle \in R$ for all $x \in X$, whence, if $w^{\prime} \in R(X)$, using the Euclidean property again, $\left\langle w, w^{\prime}\right\rangle \in R$. Thus $R$ is total on $R(X)$. Finally, we show that $R(R(X)) \subseteq R(X)$. Suppose $w \in R(R(X))$, so that 
$\left\langle w^{\prime}, w\right\rangle \in R$ for some $w^{\prime} \in R(X)$. Pick any $x \in X$. Since $R$ is total on $R(X) \supseteq X,\left\langle w^{\prime}, x\right\rangle \in R$, and so, by the Euclidean property, $\langle x, w\rangle \in R$. Thus, $R(R(X)) \subseteq R(X)$, proving the claim.

For the second statement of the lemma, putting $X=$ $R\left(w_{0}\right)$ in the claim of the previous paragraph, we have $R\left(R\left(w_{0}\right)\right)=R\left(R\left(R\left(w_{0}\right)\right)\right)=R\left(R\left(R\left(R\left(w_{0}\right)\right)\right)\right)=\ldots$ Thus,

$$
\begin{aligned}
R^{*}\left(w_{0}\right) & =\left\{w_{0}\right\} \cup R\left(w_{0}\right) \cup R\left(R\left(w_{0}\right)\right) \cup \cdots \\
& =\left\{w_{0}\right\} \cup R\left(w_{0}\right) \cup R\left(R\left(w_{0}\right)\right) \\
& =\left\{w_{0}\right\} \cup R\left(R\left(w_{0}\right)\right),
\end{aligned}
$$

with the last step following from the first statement of the lemma.

Lemmas 1 and 2 show that, when discussing satisfiability over Euclidean frames, we may restrict attention to frames of the form $\left(W \cup\left\{w_{0}\right\}, R\right)$, where $R$ is total on $W$, $R\left(w_{0}\right) \subseteq W$, and $w_{0}$ may or may not be in $W$. Over such simple frames, any $\mathcal{G} \mathcal{M}$-formula can be translated into an equisatisfiable $\mathcal{C}^{1}$-formula. Specifically:

Lemma 3. Let $\mathcal{F} \subseteq\{\mathrm{Rfl}, \mathrm{Ser}, \mathrm{Sym}, \mathrm{Tr}\}$. Given a $\mathcal{G} \mathcal{M}$ formula $\varphi$, we can compute, in time bounded by a polynomial function of $\|\varphi\|$, a $\mathcal{C}^{1}$-formula $\alpha$ such that $\varphi$ is satisfiable over a frame in $\cap \mathcal{F} \cap$ Eucl if and only if $\alpha$ is satisfiable.

Proof: Let $q_{0}, q_{1}, q_{2}$ be new unary predicates (i.e., pairwise distinct and not in $\Pi$ ). We define a two-stage translation from $\mathcal{G M}$ into $\mathcal{C}^{1}$ as follows. Notice that the definition of $f_{1}$ makes reference to $f_{2}$, but not vice versa.

$$
\begin{aligned}
f_{1}(p) & =p(x) & & \text { (for } p \in \Pi) \\
f_{1}(\varphi \wedge \psi) & =f_{1}(\varphi) \wedge f_{1}(\psi) & & \text { (sim. for } \neg, \vee, \text { etc.) } \\
f_{1}\left(\diamond_{\geq C} \varphi\right) & =\exists_{\geq C} \cdot x\left(f_{2}(\varphi) \wedge q_{1}(x)\right) & & \\
f_{1}\left(\diamond_{\leq C} \varphi\right) & =\exists_{\leq C} x \cdot\left(f_{2}(\varphi) \wedge q_{1}(x)\right) & & \\
f_{2}(p) & =p(x) & & \text { (for } p \in \Pi) \\
f_{2}(\varphi \wedge \psi) & =f_{2}(\varphi) \wedge f_{2}(\psi) & & \text { (sim. for } \neg, \vee, \text { etc.) } \\
f_{2}\left(\diamond_{\geq C} \varphi\right) & =\exists_{\geq C} x \cdot\left(f_{2}(\varphi) \wedge q_{2}(x)\right) & & \\
f_{2}\left(\diamond_{\leq C} \varphi\right) & =\exists_{\leq C} x .\left(f_{2}(\varphi) \wedge q_{2}(x)\right) . & &
\end{aligned}
$$

Next, we define first-order formulas (in fact, $\mathcal{C}^{1}$-formulas), which, for Euclidean frames, act as substitutes for the conditions of reflexivity, seriality, symmetry and transitivity:

$$
\begin{aligned}
\varepsilon_{\mathrm{Rfl}} & =\forall x \cdot\left(q_{0}(x) \rightarrow q_{1}(x)\right) \\
\varepsilon_{\mathrm{Ser}} & =\exists x \cdot q_{1}(x) \\
\varepsilon_{\mathrm{Sym}} & =\forall x \cdot\left(q_{0}(x) \rightarrow q_{1}(x)\right) \vee \neg \exists x \cdot q_{1}(x) \\
\varepsilon_{\mathrm{Tr}} & =\forall x \cdot\left(q_{2}(x) \rightarrow q_{1}(x)\right) .
\end{aligned}
$$

Let us define the required $\mathcal{C}^{1}$ formula $\alpha$ as follows:

$$
\alpha=\exists x \cdot\left(f_{1}(\varphi) \wedge q_{0}(x)\right) \wedge \forall x .\left(q_{1}(x) \rightarrow q_{2}(x)\right) \wedge \bigwedge_{\mathcal{K} \in \mathcal{F}} \varepsilon_{\mathcal{K}} .
$$

Clearly, $\alpha$ can be constructed in polynomial time from $\varphi$. It remains to demonstrate that $\varphi$ is satisfiable over a frame in $\bigcap \mathcal{F} \cap$ Eucl if and only if $\alpha$ is satisfiable.

Suppose $\mathfrak{A} \models w_{0} \varphi$, where $\mathfrak{A}$ is a structure over a Euclidean frame $(W, R)$. Let $\mathfrak{B}$ be the substructure generated by $\left\{w_{0}\right\}$-in other words, the restriction of $\mathfrak{A}$ to $R^{*}\left(w_{0}\right)$. By Lemma $1 \mathfrak{B} \models w_{0} \varphi$. Expand $\mathfrak{B}$ to a structure $\mathfrak{B}^{+}$by setting

$$
q_{0}^{\mathfrak{B}^{+}}=\left\{w_{0}\right\}, \quad q_{1}^{\mathfrak{B}^{+}}=R\left(w_{0}\right), \quad q_{2}^{\mathfrak{B}^{+}}=R\left(R\left(w_{0}\right)\right) .
$$

We shall show that $\mathfrak{B}^{+} \models \alpha$. By Statement 1 of Lemma 2 $\mathfrak{B}^{+} \models \forall x \cdot\left(q_{1}(x) \rightarrow q_{2}(x)\right)$. Using Lemma 2, a structural induction on $\psi$ easily establishes the following condition.

For all $w \in q_{2}^{\mathfrak{B}^{+}}$, and all $\mathcal{G} \mathcal{M}$-formulas $\psi$,

$$
\mathfrak{B} \models{ }_{w} \psi \text { if and only if } \mathfrak{B}^{+} \models f_{2}(\psi)[w] \text {. }
$$

Using (1), a further structural induction establishes the following condition.

For all $\mathcal{G M}$-formulas $\psi$,

$$
\mathfrak{B} \models w_{0} \psi \text { if and only if } \mathfrak{B}^{+} \models f_{1}(\psi)\left[w_{0}\right] \text {. }
$$

From (2), it follows that $\mathfrak{B}^{+} \models \exists x\left(f_{1}(\varphi) \wedge q_{0}(x)\right)$. It remains to show that, for all $\mathcal{K} \in\{\mathrm{Rfl}, \mathrm{Ser}, \mathrm{Sym}, \mathrm{Tr}\}$, $(W, R) \in \mathcal{K}$ implies $\mathfrak{B}^{+} \models \varepsilon_{\mathcal{K}}$. Suppose, then $(W, R) \in \mathcal{K}$; we consider the four cases in turn.

1) If $\mathcal{K}=\mathrm{Rfl}$, then $w_{0} \in R\left(w_{0}\right)$. It follows that $\mathfrak{B}^{+} \models \forall x .\left(q_{0}(x) \rightarrow q_{1}(x)\right)$.

2) If $\mathcal{K}=\operatorname{Ser}$, then $R\left(w_{0}\right) \neq \emptyset$. It follows that $\mathfrak{B}^{+} \models \exists x \cdot q_{1}(x)$.

3) If $\mathcal{K}=$ Sym, then, since $(W, R)$ is both symmetric and Euclidean, either $\left\langle w_{0}, w_{0}\right\rangle \in R$, or $R\left(w_{0}\right)=\emptyset$. Thus, either $\mathfrak{B}^{+} \models \forall x .\left(q_{0}(x) \rightarrow q_{1}(x)\right)$, or $\mathfrak{B}^{+} \models \forall x . \neg q_{1}(x)$.

4) If $\mathcal{K}=\operatorname{Tr}$, then $R\left(R\left(w_{0}\right)\right) \subseteq R\left(w_{0}\right)$. It follows that $\mathfrak{B}^{+} \models \forall x .\left(q_{2}(x) \rightarrow q_{1}(x)\right)$.

This establishes that $\mathfrak{B}^{+} \models \alpha$, as required.

Conversely, suppose $\mathfrak{A} \models \alpha$, where $\mathfrak{A}$ interprets $\Sigma$ together with the predicates $q_{0}, q_{1}$ and $q_{2}$. Let $\mathfrak{B}^{+}$be the substructure of $\mathfrak{A}$ with domain $W=q_{0}^{\mathfrak{A}} \cup q_{1}^{\mathfrak{A}} \cup q_{2}^{\mathfrak{A}}$, and let $w_{0} \in W$ be some element satisfying $f_{1}(\varphi) \wedge q_{0}(x)$. Since all quantification in $f_{1}(\varphi)$ is limited to elements satisfying $q_{1}$ or $q_{2}, \mathfrak{B}^{+} \models \alpha$; and since $\alpha$ contains no occurrences of $r$, we may without loss of generality assume that

$$
r^{\mathfrak{B}^{+}}=\left(q_{0}^{\mathfrak{B}^{+}} \times q_{1}^{\mathfrak{B}^{+}}\right) \cup\left(q_{2}^{\mathfrak{B}^{+}} \times q_{2}^{\mathfrak{B}^{+}}\right) .
$$

Let $\mathfrak{B}$ be the $\Sigma$-reduct of $\mathfrak{B}^{+}$obtained by ignoring the predicates $q_{0}, q_{1}$ and $q_{2}$; and let $R=r^{\mathfrak{B}^{+}}$, so that $\mathfrak{B}$ is a structure over the frame $(W, R)$. We show that $\mathfrak{B} \vDash \models_{w_{0}} \varphi$, and, moreover, $(W, R) \in \bigcap \mathcal{F} \cap$ Eucl. Using the definition of $r^{\mathfrak{B}^{+}}$ in (3), two simple structural inductions again establish (1), and thence (2). And from (2), it follows that $\mathfrak{B} \models w_{0} \varphi$. It remains to show that, for all $\mathcal{K} \in\{\mathrm{Rfl}, \mathrm{Ser}, \mathrm{Sym}, \mathrm{Tr}\}$, 
$\mathfrak{B}^{+} \models \varepsilon_{\mathcal{K}}$ implies $(W, R) \in \mathcal{K}$. Suppose, then $\mathfrak{B}^{+} \models \varepsilon_{\mathcal{K}}$; we consider the four cases in turn, making implicit use of (3) throughout. Note also that, since $\mathfrak{B}^{+} \models \alpha, q_{1}^{\mathfrak{B}^{+}} \subseteq q_{2}^{\mathfrak{B}^{+}}$.

1) If $\mathcal{K}=\mathrm{Rfl}, q_{0}^{\mathfrak{B}^{+}} \subseteq q_{1}^{\mathfrak{B}^{+}} \subseteq q_{2}^{\mathfrak{B}^{+}}$, whence $(W, R)$ is total, and hence certainly reflexive.

2) If $\mathcal{K}=\operatorname{Ser}$, then $q_{1}^{\mathfrak{B}^{+}} \neq \emptyset$, whence $(W, R)$ is visibly serial.

3) If $\mathcal{K}=$ Sym, either $q_{0}^{\mathfrak{B}^{+}} \subseteq q_{1}^{\mathfrak{B}^{+}} \subseteq q_{2}^{\mathfrak{B}^{+}}$or $q_{1}^{\mathfrak{B}^{+}}=\emptyset$. In the former case, $(W, R)$ is total, and hence certainly symmetric; in the latter, $(W, R)$ is visibly symmetric.

4) If $\mathcal{K}=\operatorname{Tr}$, then $q_{2}^{\mathfrak{B}^{+}} \subseteq q_{1}^{\mathfrak{B}^{+}}$, whence $(W, R)$ is visibly transitive.

The upper bound of Theorem 4 now follows by Theorem 6 and Lemma 3, since Sym $\cap \operatorname{Tr} \subseteq$ Eucl. The lower bound is trivial, since $\mathcal{G M}$ includes propositional logic.

\section{TRANSITIVE FRAMES}

The purpose of this section is to establish Theorem 5 The upper bound (Section IV-A) is obtained by proving that every $\mathcal{G M}$-formula $\varphi$ that is satisfiable over a transitive (transitive and reflexive) frame is also satisfiable over a transitive (transitive and reflexive) frame whose size is bounded by an exponential function of $\|\varphi\|$. It is shown in [8] that every $\mathcal{G M}$-formula satisfiable over a transitive frame is also satisfiable over a finite transitive frame. However, this paper gives no bound on the size of the satisfying structure. The matching lower bound (Section IV-B) is obtained by a reduction from exponential tiling problems. Interestingly, this reduction features only formulas in which all numerical subscripts are bounded by 1 . Thus, the lower complexitybound of Theorem 5 continues to hold even under unary coding of numerical subscripts.

One note on terminology before we proceed. In the context of (graded) modal logic, it is customary to think of the unary predicates in $\Pi$ as proposition letters, because they receive truth-values relative to worlds. Since we shall not be concerned with $\mathcal{C}^{1}$ or other first-order fragments in the sequel, we adopt this practice from now on. Accordingly, a propositional formula is one containing no modal operators. Finally, we shall relax our stance on valuations, allowing structures to interpret only those proposition letters involved in some collection of formulas of interest, rather than every proposition letter in $\Pi$.

\section{A. Membership in NExpTime}

First we demonstrate that every $\mathcal{G M}$-formula can be transformed into a normal form preserving satisfiability over transitive frames. This normal form is broadly similar to the so-called Scott normal form for the two-variable fragment of first-order logic, and is likewise obtained by a straightforward renaming procedure. For the next lemma, recall that $\square \varphi$ abbreviates $\varphi \wedge \square \varphi$.
Lemma 4. Let $\varphi$ be a $\mathcal{G M - f o r m u l a . ~ W e ~ c a n ~ c o m p u t e , ~ i n ~}$ time bounded by a polynomial function of $\|\varphi\|$, a $\mathcal{G M}$ formula $\psi$ of the form

$$
\eta \wedge \boxminus\left(\theta \wedge \bigwedge_{1 \leq i \leq \ell}\left(p_{i} \rightarrow \diamond_{\geq C_{i}} \pi_{i}\right) \wedge \bigwedge_{1 \leq j \leq m}\left(q_{j} \rightarrow \diamond_{\leq D_{j}} \chi_{j}\right)\right)
$$

where the $p_{i}$ and the $q_{j}$ are proposition letters, the $C_{i}$ and $D_{j}$ are natural numbers, and $\eta, \theta$, the $\pi_{i}$ and the $\chi_{j}$ are propositional formulas, such that $\varphi$ and $\psi$ are satisfiable over exactly the same transitive frames.

Proof: As usual, if $\rho$ is a subformula of $\varphi$ and $\sigma$ a formula, we denote by $\varphi[\sigma / \rho]$ the result of substituting $\sigma$ for every occurrence of $\rho$ in $\varphi$. If $\rho$ is a formula of the form $\diamond_{\leq C} \pi$, denote by $\bar{\rho}$ the corresponding formula $\diamond_{\geq(C+1)} \pi$; similarly, if $\rho$ is a formula of the form $\diamond_{\geq C} \pi$, with $C>0$, denote by $\bar{\rho}$ the corresponding formula $\diamond_{\leq(C-1)} \pi$.

We may assume that $\varphi$ contains no subformulas of the form $\diamond_{\geq 0} \pi$, since these may be replaced with any tautology. Suppose $\varphi$ is not propositional, and let $\rho$ be any subformula of $\varphi$ having either of the forms $\diamond_{\leq C} \pi$ or $\diamond_{\geq C} \pi$, with $\pi$ propositional. (In the latter case, $C>0$.) Let $p$ and $q$ be fresh proposition letters, and let $\varphi^{\prime}$ be the formula

$$
\varphi[p / \rho] \wedge \sqcup(p \vee q) \wedge \sqcup(p \rightarrow \rho) \wedge \sqcup(q \rightarrow \bar{\rho}) .
$$

It is easy to verify that, if $\mathfrak{A} \models_{w} \varphi^{\prime}$ with $\mathfrak{A}$ transitive, then $\mathfrak{A} \models_{w} \varphi$. Conversely, if $\mathfrak{A} \models_{w_{0}} \varphi$, we may expand $\mathfrak{A}$ to a structure $\mathfrak{A}^{\prime}$ by setting $\mathfrak{A}^{\prime} \models_{w} p$ if and only if $\mathfrak{A}^{\prime} \models_{w}$ $\rho$ and $\mathfrak{A}^{\prime} \models_{w} q$ if and only if $\mathfrak{A}^{\prime} \not \nvdash_{w} \rho$, for all worlds $w$ : evidently, $\mathfrak{A}^{\prime} \models \models_{w_{0}} \varphi^{\prime}$. Thus, $\varphi$ and $\varphi^{\prime}$ are satisfiable over the same transitive frames. Repeating this process and re-grouping conjuncts eventually leads to a formula of the form (4) as required.

We next present lemmas describing transformations of transitive structures, in which we use the following terminology. Let $\mathfrak{A}=\langle W, R, V\rangle$ be a transitive structure, and $w_{1}, w_{2}$ be worlds of $W$. We say: $w_{2}$ is an $R$-successor of $w_{1}$ if $\left\langle w_{1}, w_{2}\right\rangle \in R ; w_{2}$ is a strict $R$-successor of $w_{1}$ if $\left\langle w_{1}, w_{2}\right\rangle \in R$, but $\left\langle w_{2}, w_{1}\right\rangle \notin R ; w_{1}$ and $w_{2}$ are $R$ equivalent if $\left\langle w_{1}, w_{2}\right\rangle \in R$ and $\left\langle w_{2}, w_{1}\right\rangle \in R$. The $R$-clique for $w_{1}$ in $\mathfrak{A}$ is the set $Q_{\mathfrak{A}}\left(w_{1}\right) \subseteq W$ consisting of $w_{1}$ and all worlds $R$-equivalent to $w_{1}$. We say that $w_{2}$ is a direct $R$-successor of $w_{1}$ if $w_{2}$ is a strict $R$-successor of $w_{1}$ and, for every $w \in W$ such that $\left\langle w_{1}, w\right\rangle \in R$ and $\left\langle w, w_{2}\right\rangle \in R$, we have either $w \in Q_{\mathfrak{A}}\left(w_{1}\right)$ or $w \in Q_{\mathfrak{A}}\left(w_{2}\right)$.

The depth of a structure $\mathfrak{A}$ is the maximum over all $k \geq 0$ for which there exist worlds $w_{0}, \ldots, w_{k} \in W$ such that $w_{i}$ is a strict $R$-successor of $w_{i-1}$ for every $i$ with $1 \leq i \leq$ $k$, or $\infty$ if no such maximum exists. The breadth of $\mathfrak{A}$ is the maximum over all $k \geq 0$ for which there exist worlds $w, w_{1}, \ldots, w_{k}$ such that $w_{i}$ is a direct $R$-successor of $w$ for every $i$ with $1 \leq i \leq k$, and the sets $Q_{\mathfrak{A}}\left(w_{1}\right), \ldots, Q_{\mathfrak{A}}\left(w_{k}\right)$ are disjoint, or $\infty$ if no such maximum exists. The width of $\mathfrak{A}$ is the smallest $k$ such that $k \geq\left\|Q_{\mathfrak{A}}(w)\right\|$ for all $w \in W$, or $\infty$ if no such $k$ exists. 
Lemma 5. Let $\mathfrak{A}$ be a structure of depth $d$, breadth $b$ and width $c$ (all finite), and let $w$ be a world of $\mathfrak{A}$. Then the substructure of $\mathfrak{A}$ generated by $\{w\}$ contains no more than $n$ worlds, where $n=c$ if $b=0, n=c \cdot(d+1)$ if $b=1$, and $n=c \cdot\left(b^{d+1}-1\right) /(b-1)$ otherwise.

Proof: Elementary.

We employ the following notation. For a structure $\mathfrak{A}=$ $(W, R, V)$ and a binary relation $R^{\prime}$ on $W$ (possibly different from $R$ ), we denote by $R_{\mathfrak{A}}^{\prime}(w, \varphi)$ the set $\{v \mid$ $\left.\langle w, v\rangle \in R^{\prime}, \mathfrak{A} \models_{v} \varphi\right\}$. Thus, $\mathfrak{A} \models_{w} \diamond_{\geq C} \varphi$ if and only if $\left\|R_{\mathfrak{A}}(w, \varphi)\right\| \geq C$, where $\|S\|$ denotes the cardinality of the set $S$. Similarly, $\mathfrak{A} \models_{w} \diamond_{\leq C} \varphi$ if and only if $\left\|R_{\mathfrak{A}}(w, \varphi)\right\| \leq C$.

Lemma 6. Let $\varphi$ be a formula of the form (4). If $\varphi$ has a transitive model $\mathfrak{A}$, then it has a transitive model $\mathfrak{A}^{\prime}$ with depth $d^{\prime} \leq 2 \ell$, breadth $b^{\prime} \leq \sum_{i=1}^{\ell} C_{i}$ and width $c^{\prime} \leq \sum_{i=1}^{\ell} C_{i}+1$. If $\mathfrak{A}$ is reflexive, then we can additionally ensure that $\mathfrak{A}^{\prime}$ is also reflexive.

Proof: Let $\mathfrak{A}=(W, R, V)$. We construct $\mathfrak{A}^{\prime}=$ $\left(W^{\prime}, R^{\prime}, V^{\prime}\right)$ from $\mathfrak{A}$ in four stages.

Stage 1: Adapting a technique employed in [8] to establish the finite model property for $\mathcal{G M}$-formulas, we first define a transitive model $\mathfrak{A}^{\prime}$ of $\varphi$, reflexive if $\mathfrak{A}$ is, such that $\mathfrak{A}^{\prime}$ has finite depth. The strategy is to enlarge the relation $R$ (thus reducing the number of strict successors of worlds in $W$ ), preserving satisfaction for subformulas of the form $\diamond_{\leq D_{j}} \chi_{j}$.

For $w \in W$ define $d_{\mathfrak{A}}^{j}(w):=\min \left(D_{j}+1,\left\|R^{*}\left(w, \chi_{j}\right)\right\|\right)$ where $D_{j}$ and $\chi_{j}(1 \leq j \leq m)$ are as in (4), and $R^{*}$ is the reflexive closure of $R$. Let $R_{d}:=\left\{\left\langle w_{1}, w_{2}\right\rangle \in R \mid\right.$ $\left.d_{\mathfrak{A}}^{j}\left(w_{1}\right)=d_{\mathfrak{A}}^{j}\left(w_{2}\right), 1 \leq j \leq m\right\}$ be the restriction of $R$ to pairs of elements that have the same values of $d_{\mathfrak{A}}^{j}(w)$, and let $R_{d}^{-}:=\left\{\left\langle w_{1}, w_{2}\right\rangle \mid\left\langle w_{2}, w_{1}\right\rangle \in R_{d}\right\}$ be the inverse of $R_{d}$. Let $\mathfrak{A}^{\prime}=\left(W, R^{\prime}, V\right)$ be obtained from $\mathfrak{A}=(W, R, V)$ by setting $R^{\prime}:=\left(R \cup R_{d}^{-}\right)^{+}$. Intuitively, if $w_{1}$ is $R$-reachable from $w_{2}$, and, for all $j(1 \leq j \leq m), w_{1}$ and $w_{2}$ agree on the number (up to the limit of $D_{j}$ ) of $\chi_{j}$-worlds that are $R$-reachable from them, then we make $w_{1}$ and $w_{2} R^{\prime}$ equivalent. We show that $\mathfrak{A}^{\prime}$ satisfies $\varphi$, is reflexive if $\mathfrak{A}$ is, and has finite depth.

Since $R \subseteq R^{\prime}, \mathfrak{A}^{\prime}$ is reflexive if $\mathfrak{A}$ is. We claim that $\mathfrak{A}^{\prime}$ has finite depth. Indeed, for every $w_{1}, w_{2} \in W$ such that $w_{2}$ is a strict $R^{\prime}$-successor of $w_{1}$, we have $d_{\mathfrak{A}}^{j}\left(w_{1}\right) \geq d_{\mathfrak{A}}^{j}\left(w_{2}\right)$ for all $j$, and $d_{\mathfrak{A}}^{j}\left(w_{1}\right)>d_{\mathfrak{A}}^{j}\left(w_{2}\right)$ for some $j(1 \leq j \leq m)$. Hence $\sum_{j=1}^{m} d_{\mathfrak{A}}^{j}\left(w_{1}\right)>\sum_{j=1}^{m} d_{\mathfrak{A}}^{j}\left(w_{2}\right)$. Since $d_{\mathfrak{A}}^{j}(w) \leq D_{j}+1$ for every $w \in W$ and every $j(1 \leq j \leq m)$, the length of every chain $w_{0}, \ldots, w_{k}$ such that $w_{i}$ is a strict $R^{\prime}$-successor of $w_{i-1}(1 \leq i \leq k)$, is bounded by $\sum_{j=1}^{m} D_{j}+m$.

In order to prove that $\mathfrak{A}^{\prime}$ satisfies $\varphi$, we first prove that $d_{\mathfrak{A}}^{j}(w)=d_{\mathfrak{A}^{\prime}}^{j}(w)$ for every $w \in W$ and every $j$ $(1 \leq j \leq m)$. Assume to the contrary that $d_{\mathfrak{A}}^{j}(w) \neq d_{\mathfrak{A}^{\prime}}^{j}(w)$ for some $w \in W$ and some $j(1 \leq j \leq m)$. Since $R \subseteq R^{\prime}$, we have $d_{\mathfrak{A}}^{j}(w)<d_{\mathfrak{A}^{\prime}}^{j}(w) \leq D_{j}+1$, which means, in particular, that there exists an element $w^{\prime} \in W$ with $\mathfrak{A} \models_{w^{\prime}} \chi_{j}$ such that $\left\langle w, w^{\prime}\right\rangle \in R^{\prime}$ but $\left\langle w, w^{\prime}\right\rangle \notin R$.

Since $\left\langle w, w^{\prime}\right\rangle \in R^{\prime}$, by definition of $R^{\prime}$, there exists a sequence $w_{0}, \ldots, w_{k}$ of different worlds in $W$ such that $w_{0}=w, w_{k}=w^{\prime}$, and $\left\langle w_{i-1}, w_{i}\right\rangle \in R \cup R_{d}^{-}$for every $i(1 \leq i \leq k)$. Note that $d_{\mathfrak{A}}^{j}\left(w_{i-1}\right) \geq d_{\mathfrak{A}}^{j}\left(w_{i}\right)$ for every $i(1 \leq i \leq k)$ and every $j(1 \leq j \leq m)$. Take the maximal $i(1 \leq i \leq k)$ such that $\left\langle w_{i-1}, w^{\prime}\right\rangle \notin R$. Since $\left\langle w_{0}, w^{\prime}\right\rangle=\left\langle w, w^{\prime}\right\rangle \notin R$, such a maximal $i$ always exists. Then $\left\langle w_{i}, w^{\prime}\right\rangle \in R^{*}$, and $\left\langle w_{i-1}, w_{i}\right\rangle \notin R$. Since $\left\langle w_{i-1}, w_{i}\right\rangle \in R \cup R_{d}^{-}$, we have $\left\langle w_{i-1}, w_{i}\right\rangle \in R_{d}^{-}$, and so $d_{\mathfrak{A}}^{j}\left(w_{i-1}\right)=d_{\mathfrak{A}}^{j}\left(w_{i}\right)$ by definition of $R_{d}$. Since $d_{\mathfrak{A}}^{j}\left(w_{i}\right) \leq$ $d_{\mathfrak{A}}^{j}\left(w_{0}\right)=d_{\mathfrak{A}}^{j}(w)<D_{j}+1$, we obtain a contradiction, due to the fact that $d_{\mathfrak{A}}^{j}\left(w_{i-1}\right)=d_{\mathfrak{A}}^{j}\left(w_{i}\right) \leq D_{j},\left\langle w_{i-1}, w^{\prime}\right\rangle \notin R^{*}$, $\left\langle w_{i}, w^{\prime}\right\rangle \in R^{*}$, and $\mathfrak{A} \models_{w^{\prime}} \chi_{j}$.

Now to complete the proof that $\mathfrak{A}^{\prime}$ satisfies $\varphi$, we demonstrate that, if $\psi$ is any of the formulas $\eta, \theta,\left(p_{i} \rightarrow \diamond_{C_{i}} \pi\right)$ or $\left(q_{j} \rightarrow \diamond_{\leq D_{j}} \chi_{j}\right)$ occurring in (4), and $w \in W$, then $\mathfrak{A} \models_{w} \psi$ implies $\mathfrak{A}^{\prime} \models_{w} \psi$. Indeed, for the propositional subformulas $\eta$ and $\theta$, this is immediate. For subformulas $p_{i} \rightarrow \diamond_{\geq C_{i}} \pi_{i}$, this holds since $R \subseteq R^{\prime}$. Finally, for subformulas $q_{j} \rightarrow \diamond_{\leq D_{j}} \chi_{j}$ this follows from the property $d_{\mathfrak{A}}^{j}(w)=d_{\mathfrak{A}^{\prime}}^{j}(w)$.

Stage 2: By Stage 1, we may assume that $\mathfrak{A}$ has finite depth $d$. We define a transitive model $\mathfrak{A}^{\prime}$ of $\varphi$, reflexive if $\mathfrak{A}$ is, such that $\mathfrak{A}^{\prime}$ has depth $d^{\prime} \leq 2 \ell$. If $d \leq 2 \ell$ then we take $\mathfrak{A}^{\prime}=\mathfrak{A}$. Otherwise, we obtain $\mathfrak{A}^{\prime}$ from $\mathfrak{A}$ by contracting the relation $R$ (removing unnecessary direct successors of worlds in $W$ ), preserving satisfaction for subformulas of the form $\diamond_{\geq C_{i}} \pi_{i}$. Define, for every $w \in W$, two sets of indices:

$$
\begin{aligned}
& I_{\mathfrak{A}}(w)=\left\{i \mid 1 \leq i \leq \ell,\left\|R\left(w, \pi_{i}\right)\right\| \geq C_{i}\right\}, \text { and } \\
& I_{\mathfrak{A}}^{s}(w)=\left\{i \mid 1 \leq i \leq \ell,\left\|R\left(w, \pi_{i}\right) \backslash Q_{\mathfrak{A}}(w)\right\| \geq C_{i}\right\},
\end{aligned}
$$

where $\pi_{i}$ and $C_{i}$ are as in (4), $1 \leq i \leq \ell$. Note that:

(P1) $I_{\mathfrak{A}}^{s}(w) \subseteq I_{\mathfrak{A}}(w)$ for every $w \in W$, and

(P2) $I_{\mathfrak{A}}\left(w_{2}\right) \subseteq I_{\mathfrak{A}}^{s}\left(w_{1}\right)$ if $w_{2}$ is a strict $R$-successor of $w_{1}$.

Define the structure $\mathfrak{A}^{\prime}=\left\langle W, R^{\prime}, V\right\rangle$ by setting

$$
\begin{gathered}
R^{\prime}:=R \backslash\left\{\left\langle w_{1}, w_{2}\right\rangle \mid w_{2} \text { is a direct } R^{\prime} \text {-successor of } w_{1}\right. \\
\text { and } \left.I_{\mathfrak{A}}^{s}\left(w_{2}\right)=I_{\mathfrak{A}}\left(w_{1}\right)\right\} .
\end{gathered}
$$

We claim that $\mathfrak{A}^{\prime}$ is a transitive structure which satisfies $\varphi$, is reflexive if $\mathfrak{A}$ is, and has depth $d^{\prime}<d$. Repeating this step sufficiently often, we eventually ensure that $d^{\prime} \leq 2 \ell$.

It is easy to see that $R^{\prime}$ is transitive if $R$ is transitive. Indeed, if $\left\langle w_{1}, w_{2}\right\rangle \in R^{\prime}$ and $\left\langle w_{2}, w_{3}\right\rangle \in R^{\prime}$, we have $\left\langle w_{1}, w_{3}\right\rangle \in R$, and either $(i) w_{3}$ is not a direct $R$-successor of $w_{1}$, or $(i i) w_{2} \in Q_{\mathfrak{A}}\left(w_{1}\right)$ and $I_{\mathfrak{A}}^{s}\left(w_{3}\right) \neq I_{\mathfrak{A}}\left(w_{2}\right)=$ $I_{\mathfrak{A}}\left(w_{1}\right)$, or $(i i i) w_{2} \in Q_{\mathfrak{A}}\left(w_{3}\right)$ and $I_{\mathfrak{A}}^{s}\left(w_{3}\right)=I_{\mathfrak{A}}^{s}\left(w_{2}\right) \neq$ $I_{\mathfrak{A}}\left(w_{1}\right)$. In all of these three cases, we have $\left\langle w_{1}, w_{3}\right\rangle \in R^{\prime}$ by the definition of $R^{\prime}$. Trivially, $R^{\prime}$ is reflexive if $R$ is.

In order to prove that $\mathfrak{A}^{\prime}$ satisfies $\varphi$, we first point out some other properties of $I_{\mathfrak{A}}(w), I_{\mathfrak{A}}^{s}(w), I_{\mathfrak{A}^{\prime}}(w)$, and $I_{\mathfrak{A}^{\prime}}^{s}(w)$ :

(P3) $\quad I_{\mathfrak{A}^{\prime}}(w) \subseteq I_{\mathfrak{A}}(w)$ and $I_{\mathfrak{A}^{\prime}}^{s}(w) \subseteq I_{\mathfrak{A}}^{s}(w)$ for $w \in W$; 
(P4) $I_{\mathfrak{A}}^{s}\left(w_{2}\right) \subseteq I_{\mathfrak{A}^{\prime}}\left(w_{1}\right)$ if $w_{2}$ is a strict $R$-successor of $w_{1}$; (P5) $I_{\mathfrak{A}^{\prime}}(w)=I_{\mathfrak{A}}(w)$ for $w \in W$.

Property (P3) holds since $R^{\prime} \subseteq R$. Property (P4) holds since, for every $i(1 \leq i \leq \ell)$, every $w_{3} \in R_{\mathfrak{A}}\left(w_{2}, \pi_{i}\right) \backslash$ $Q_{\mathfrak{A}}\left(w_{2}\right)$ is a strict non-direct $R$-successor of $w_{1}$. Hence $\left\langle w_{1}, w_{3}\right\rangle \in R^{\prime}$ by the definition of $R^{\prime}$, and so, $w_{3} \in$ $R_{\mathfrak{A}^{\prime}}\left(w_{1}, \pi_{i}\right)$. In order to prove $(\mathbf{P 5})$, by $(\mathbf{P 3})$, it suffices to prove $I_{\mathfrak{A}^{\prime}}(w) \supseteq I_{\mathfrak{A}}(w)$. Assume to the contrary that there exists $w \in W$ and $i(1 \leq i \leq \ell)$ such that $\mathfrak{A} \models_{w^{\prime}} \pi_{i}$ (equivalently, $\left.\mathfrak{A}^{\prime} \models_{w^{\prime}} \pi_{i}\right),\left\langle w, w^{\prime}\right\rangle \in R$, and $\left\langle w, w^{\prime}\right\rangle \notin R^{\prime}$. By the definition of $R^{\prime}$, this is only possible if $w^{\prime}$ is a direct $R$ successor of $w$ and $I_{\mathfrak{A}}^{s}\left(w^{\prime}\right)=I_{\mathfrak{A}}(w)$. But then, by (P4), we have $I_{\mathfrak{A}}^{s}\left(w^{\prime}\right) \subseteq I_{\mathfrak{A}^{\prime}}(w)$. Hence $I_{\mathfrak{A}}(w)=I_{\mathfrak{A}}^{s}\left(w^{\prime}\right) \subseteq I_{\mathfrak{A}^{\prime}}(w)$, which contradicts the assumption that $I_{\mathfrak{A}}(w) \backslash I_{\mathfrak{A}^{\prime}}(w) \neq \emptyset$.

In order to prove that $\mathfrak{A}^{\prime}$ satisfies $\varphi$, it is sufficient, as in Stage 1, to demonstrate that, if $\psi$ is any of the formulas $\eta$, $\theta,\left(p_{i} \rightarrow \diamond_{C_{i}} \pi_{i}\right)$ or $\left(q_{j} \rightarrow \diamond_{\leq D_{j}} \chi_{j}\right)$ occurring in (4), and $w \in W$, then $\mathfrak{A} \models_{w} \psi$ implies $\mathfrak{A}^{\prime} \models_{w} \psi$. This property holds for $\psi=\eta, \psi=\theta$, and $\psi=\left(q_{j} \rightarrow \diamond_{\leq D_{j}} \chi_{j}\right), 1 \leq$ $j \leq m$, since $R^{\prime} \subseteq R$. For $\psi=\left(p_{i} \rightarrow \diamond_{C_{i}} \pi_{i}\right), 1 \leq i \leq m$, this property holds by (P5).

Finally, it remains to demonstrate that the depth of $\mathfrak{A}^{\prime}$ is smaller than the depth $d$ of $\mathfrak{A}$. Suppose, to the contrary, that there exists a sequence of worlds $w_{0}, \ldots, w_{d}$ in $W$ such that $w_{i}$ is a strict $R^{\prime}$-successor of $w_{i-1}, 1 \leq i \leq d$. By definition of $R^{\prime}$, every $w_{i}$ is a strict $R$-successor of $w_{i-1}$, and, since $d$ is the depth of $\mathfrak{A}, w_{i}$ is in fact a direct $R$ successor of $w_{i-1}, 1 \leq i \leq d$. Again, by definition of $R^{\prime}$, we have $I_{\mathfrak{A}}^{s}\left(w_{i}\right) \neq I_{\mathfrak{A}}\left(w_{i-1}\right), 1 \leq i \leq d$. By $(\mathbf{P 1})$ and $(\mathbf{P 2})$ we have $I_{\mathfrak{A}}^{s}\left(w_{i}\right) \subsetneq I_{\mathfrak{A}}\left(w_{i-1}\right)$ and $I_{\mathfrak{A}}\left(w_{i}\right) \subseteq I_{\mathfrak{A}}^{s}\left(w_{i-1}\right)$, so $\left\|I_{\mathfrak{A}}^{s}\left(w_{i}\right)\right\|+\left\|I_{\mathfrak{A}}\left(w_{i}\right)\right\|<\left\|I_{\mathfrak{A}}^{s}\left(w_{i-1}\right)\right\|+\left\|I_{\mathfrak{A}}\left(w_{i-1}\right)\right\|, 1 \leq i \leq d$. Since $\left\|I_{\mathfrak{A}}^{s}(w)\right\| \leq\left\|I_{\mathfrak{A}}(w)\right\| \leq \ell$ for every $w$ in $W$, this is possible only if $d \leq 2 \ell$.

Stage 3: By Stage 2, we may assume that $\mathfrak{A}$ has depth $d \leq 2 \ell$. We define a transitive model $\mathfrak{A}^{\prime}$ of $\varphi$, reflexive if $\mathfrak{A}$ is, such that $\mathfrak{A}^{\prime}$ has depth $d^{\prime} \leq 2 \ell$ and breadth $b^{\prime} \leq \sum_{i=1}^{\ell} C_{i}$. For every element $w \in W$ and every $i$ with $1 \leq i \leq \ell$, let $W_{i}(w)$ be the set of strict $R$-successors of $w$ for which $\pi_{i}$ holds. We call the elements of $W_{i}(w)$ the strict $\pi_{i}$-witnesses for $w$. Note that $W_{i}\left(w_{1}\right)=W_{i}\left(w_{2}\right)$ when $w_{1}$ and $w_{2}$ are $R$-equivalent. Let $W_{i}^{\prime}(w)$ be $W_{i}(w)$ if $\left\|W_{i}(w)\right\| \leq C_{i}$ or, otherwise, a subset of $W_{i}(w)$ which contains exactly $C_{i}$ elements. We call $W_{i}^{\prime}(w)$ the selected strict $\pi_{i}$-witnesses for $w$. We assume that $W_{i}^{\prime}\left(w_{1}\right)=W_{i}^{\prime}\left(w_{2}\right)$ when $w_{1}$ and $w_{2}$ are $R$-equivalent. Let $R_{q}:=\left\{\left\langle w, w^{\prime}\right\rangle \in R \mid w^{\prime} \in Q_{\mathfrak{A}}(w)\right\}$ be the restriction of $R$ to elements of the same clique, and $R_{i}^{\prime}=\left\{\left\langle w, w^{\prime}\right\rangle \in R \mid w^{\prime} \in W_{i}^{\prime}(w)\right\}$ be the relation between an element $w \in W$ and the selected strict $\pi_{i}$-witnesses for $w$. Define the structure $\mathfrak{A}^{\prime}=\left(W, R^{\prime}, V\right)$ by setting $R^{\prime}:=$ $\left(R_{q} \cup \bigcup_{1 \leq i \leq \ell} R_{i}^{\prime}\right)^{+}$. Intuitively, $\mathfrak{A}^{\prime}$ is obtained from $\mathfrak{A}$ by removing all strict successor relations except those that are induced by selected strict witnesses. We show that $\mathfrak{A}^{\prime}$ has all required properties.

Note that $R^{\prime}$ is transitive, and reflexive if $R$ is reflexive.
Clearly, the depth of $\mathfrak{A}^{\prime}$ is bounded by $d$, since only strict successor relations are removed. It is also clear that the breadth of $\mathfrak{A}^{\prime}$ is bounded by $b=\sum_{i=1}^{\ell} C_{i}$, since for every $w \in W$ and every direct $R^{\prime}$-successor $w^{\prime}$ of $w$ there exists $i$ with $1 \leq i \leq \ell$ such that $Q_{\mathfrak{A}}\left(w^{\prime}\right) \cap W_{i}^{\prime}(w) \neq \emptyset$, and so the maximal number of such successors $w^{\prime}$ for which $Q_{\mathfrak{A}}\left(w^{\prime}\right)$ are disjoint is bounded by $\sum_{i=1}^{\ell}\left\|W_{i}^{\prime}(w)\right\| \leq \sum_{i=1}^{\ell} C_{i}=b$.

It remains to demonstrate that $\mathfrak{A}^{\prime}$ satisfies $\varphi$. Clearly, the set of worlds $w \in W$ that satisfy subformulas $\eta$ and $\theta$ has not changed. The set of worlds that satisfy subformulas $\left(q_{j} \rightarrow\right.$ $\left.\diamond_{\leq D_{j}} \chi_{j}\right)$ can only have increased, since $R^{\prime} \subseteq R$. Finally, the set of worlds that satisfy subformulas $\left(p_{i} \rightarrow \diamond_{\geq C_{i}} \pi_{i}\right)$ has not changed, since, for every $w \in W$, the number of direct $\pi_{i}$-witnesses has either not changed, or is at least $C_{i}$. Stage 4: By Stage 3, we may assume that $\mathfrak{A}$ has depth $d \leq 2 \ell$ and breadth $b \leq \sum_{i=1}^{\ell} C_{i}$. We define a structure $\mathfrak{A}^{\prime}$ with all the properties required by the lemma. For every element $w \in W$, and every $i$ with $1 \leq i \leq \ell$, let $Q_{i}(w)$ be the set of elements in $Q_{\mathfrak{A}}(w)$ for which $\pi_{i}$ holds. We call the elements of $Q_{i}(w)$ the equivalent $\pi_{i}$-witnesses for $w$. Note that $Q_{i}\left(w_{1}\right)=Q_{i}\left(w_{2}\right)$ when $w_{1}$ and $w_{2}$ are $R$-equivalent. Let $Q_{i}^{\prime}(w)$ be $Q_{i}(w)$ if $\left\|Q_{i}(w)\right\| \leq C_{i}$ or, otherwise, a subset of $Q_{i}(w)$ which contains exactly $C_{i}$ elements. We call $Q_{i}^{\prime}(w)$ the selected equivalent $\pi_{i}$-witnesses for $w$. Also let $Q_{0}^{\prime}(w)$ be a singleton set containing an element of $Q_{\mathfrak{A}}(w)$ that satisfies $\varphi$ if there is one, and any element of $Q_{\mathfrak{A}}(w)$ otherwise. We assume that $Q_{i}^{\prime}\left(w_{1}\right)=Q_{i}^{\prime}\left(w_{2}\right)$ when $w_{1}$ and $w_{2}$ are $R$-equivalent. Define the structure $\mathfrak{A}^{\prime}=\left\langle W^{\prime}, R^{\prime}, V^{\prime}\right\rangle$ by setting $W^{\prime}:=\bigcup_{w \in W, 0 \leq i<\ell} Q_{i}^{\prime}(w), R^{\prime}:=\left.R\right|_{W^{\prime}}$, and $V^{\prime}:=\left.V\right|_{W^{\prime}}$. Intuitively $\mathfrak{A}^{\prime}$ is obtained from $\mathfrak{A}$ by removing elements in every $R$-clique, except for those that are selected witnesses of other elements, and in such a way that the clique remains non-empty and contains at least one element satisfying $\varphi$ if there was one. (Note that, since no $R$-clique is completely obliterated by this process, $W^{\prime}$ is non-empty.) We show that $\mathfrak{A}^{\prime}$ has all required properties.

Clearly, $\mathfrak{A}^{\prime}$ is a transitive structure, and indeed is reflexive if $\mathfrak{A}$ is reflexive. Further, the depth and breadth of $\mathfrak{A}^{\prime}$ is bounded by the depth and breadth of $\mathfrak{A}$ since $\mathfrak{A}^{\prime}$ is a restriction of $\mathfrak{A}$ to a subset of $W$. It is easy to see that for every $w \in W^{\prime}, Q_{\mathfrak{A}^{\prime}}(w)=\bigcup_{0 \leq i \leq \ell} Q_{i}^{\prime}(w)$. Hence $\left\|Q_{\mathfrak{A}^{\prime}}(w)\right\| \leq \sum_{i=0}^{\ell}\left\|Q_{i}^{\prime}(w)\right\| \leq \sum_{i=1}^{\ell} C_{i}+1=c$. Therefore the width of $\mathfrak{A}^{\prime}$ is bounded by $c$.

It remains to demonstrate that $\mathfrak{A}^{\prime}$ satisfies $\varphi$. By the definition of $W^{\prime}$ there is a world $w_{0} \in W^{\prime}$ such that $\mathfrak{A} \models{ }_{w_{0}} \varphi$. Clearly $\mathfrak{A}^{\prime} \models_{w_{0}} \eta$ since $\mathfrak{A} \models{ }_{w_{0}} \eta$ and $V^{\prime}=\left.V\right|_{W^{\prime}}$. Let $w \in W$ be any world such that $\left\langle w_{0}, w\right\rangle \in R^{\prime}$. We need to demonstrate that $\left(\right.$ i) $\mathfrak{A}^{\prime} \models_{w} \theta$, (ii) $\mathfrak{A}^{\prime} \models_{w}\left(p_{i} \rightarrow \diamond_{\geq C_{i}} \pi_{i}\right)$, $1 \leq i \leq \ell$, and $($ iii $) \mathfrak{A}^{\prime} \models_{w}\left(q_{j} \rightarrow \diamond_{\leq D_{j}} \chi_{j}\right), 1 \leq j \leq m$. Cases $(i)$ and (iii) are trivially satisfied since $V^{\prime}=\left.V\right|_{W^{\prime}}$ and $R^{\prime} \subseteq R$. Case $(i i)$ is satisfied since, for every $i$ with $1 \leq i \leq \ell,\left\|R_{\mathfrak{A}}\left(w, \pi_{i}\right)\right\| \geq C_{i}$ implies $\left\|R_{\mathfrak{A}^{\prime}}^{\prime}\left(w, \pi_{i}\right)\right\| \geq C_{i}$. 
Lemma 7. Let $\mathfrak{A}=\langle W, R, V\rangle$ be a transitive structure that satisfies a formula $\varphi$ of the form (4). Then there exists a transitive structure $\mathfrak{A}^{\prime}=\left\langle W^{\prime}, R^{\prime}, V^{\prime}\right\rangle$ that satisfies $\varphi$ such that $\left\|W^{\prime}\right\| \leq(b+1) \cdot\left(b^{2 \ell+1}-1\right) /(b-1)$, where $b=\max \left(2, \sum_{i=1}^{\ell} C_{i}\right)$. Moreover, if $\mathfrak{A}$ is reflexive, then we can ensure that $\mathfrak{A}^{\prime}$ is also reflexive.

Proof: By Lemma 6, there is a transitive structure $\mathfrak{A}^{\prime}$ satisfying $\varphi$, reflexive if $\mathfrak{A}$ is, with depth, breadth, and width bounded respectively by $2 \ell, b$, and $b+1$. Let $w_{0}$ be such that $\mathfrak{A}^{\prime} \models{ }_{w_{0}} \varphi$, and consider the substructure of $\mathfrak{A}^{\prime}$ generated by $\left\{w_{0}\right\}$. The result now follows by Lemmas 1 and 5 .

We remark that the bound $(b+1) \cdot\left(b^{2 \ell+1}-1\right) /(b-1)$ obtained in Lemma 7 is at most exponential in the size of the input formula, even under binary coding of the numerical subscripts $C_{1}, \ldots, C_{\ell}$. Notice, incidentally, that this bound does not mention the subscripts $D_{1}, \ldots, D_{m}$ at all.

Corollary 1. If $\mathcal{F}$ is any of $\{\operatorname{Tr}\},\{\mathrm{Rfl}, \operatorname{Tr}\}$ or $\{\mathrm{Ser}, \operatorname{Tr}\}$, then the problem $\mathcal{G} \mathcal{M}_{\cap \mathcal{F}}$-Sat is in NExpTime.

Proof: Consider first the cases $\mathcal{F}=\{\operatorname{Tr}\}$ and $\mathcal{F}=$ $\{\operatorname{Tr}, \mathrm{Rfl}\}$. By Lemma 4, any $\mathcal{G M}$ formula $\varphi$ can be transformed in polynomial time into a formula $\psi$ of the form (4) preserving satisfiability over $\bigcap \mathcal{F}$. By Lemma $7 \psi$ is satisfiable over $\cap \mathcal{F}$ if and only if it is satisfiable over a frame in $\bigcap \mathcal{F}$ of size at most exponential in $\|\psi\|$. This last condition can be checked in non-deterministic exponential time. Finally, using Lemma 1, a formula $\varphi$ is satisfiable over Ser $\cap \operatorname{Tr}$ if and only if $\varphi \wedge \triangleright \diamond \top$ is satisfiable over $\operatorname{Tr}$, where $\top$ is any tautology.

\section{B. NExpTime-hardness}

To prove a matching lower bound, we employ the apparatus of tiling systems. A tiling system is a triple $\langle C, H, V\rangle$, where $C$ is a non-empty, finite set and $H, V$ are binary relations on $C$. The elements of $C$ are referred to as colours, and the relations $H$ and $V$ as the horizontal and vertical constraints, respectively. For any integer $N$, a tiling for $\langle C, H, V\rangle$ of size $N$ is a function $f:\{0, \ldots, N-1\}^{2} \rightarrow C$ such that, for all $i, j$ with $0 \leq i<N-1,0 \leq j \leq N-1$, the pair $\langle f(i, j), f(i+1, j)\rangle$ is in $H$ and for all $i, j$ with $0 \leq i \leq N-1,0 \leq j<N-1$, the pair $\langle f(i, j), f(i, j+1)\rangle$ is in $V$. A tiling of size $N$ is to be pictured as a colouring of an $N \times N$ square grid by the colours in $C$; the horizontal constraints $H$ thus specify which colours may appear 'to the right of' which other colours; the vertical constraints $V$ likewise specify which colours may appear 'above' which other colours. An $n$-tuple $\bar{c}$ of elements of $C$ is an initial configuration for the tiling $f$ if $\bar{c}=f(0,0), \ldots, f(n-1,0)$. An initial configuration for $f$ is to be pictured as a row of $n$ colours occupying the bottom left-hand corner of the grid.

Let $(C, H, V)$ be a tiling system and $p$ a polynomial. The exponential tiling problem $(C, H, V, p)$ is the following problem: given an $n$-tuple $\bar{c}$ from $C$, determine whether there exists a tiling for $(C, H, V)$ of size $2^{p(n)}$ with initial configuration $\bar{c}$. It is well-known that there exist exponential tiling problems which are NExpTime-complete (see, e.g. [19], pp. 242, ff.). We show how, for any class of frames $\mathcal{K}$ such that $\operatorname{Tr} \supseteq \mathcal{K} \supseteq \operatorname{Tr} \cap \mathrm{Rfl}$, any exponential tiling problem $(C, H, V, p)$ can be reduced to $\mathcal{G M}_{\mathcal{K}}$-Sat, in polynomial time.

In the sequel, we denote by $\{0,1\}^{*}$ the set of finite strings over the alphabet $\{0,1\}$; we denote the length of any $s \in$ $\{0,1\}^{*}$ by $\|s\|$; we denote the empty string by $\epsilon$; and we write $s \preceq t$ if $s$ is a (proper or improper) prefix of $t$. If $\|s\|=k$, then $s$ encodes a number in the range $\left[0,2^{k}-1\right]$ in the usual way; we follow standard practice in taking the left-most digit of $s$ to be the most significant. We equivocate freely between strings and the numbers they represent; in particular, we write $s+1$ to denote the string representing the successor of the number represented by $s$. Finally, if $s$ is a string and $1 \leq k \leq\|s\|$, denote the $k$ th element of $s$ (counting from the left and starting with 1 ) by $s[k]$. We use the notation $\pm_{i} \varphi$ (with $i$ a numerical subscript), to stand, ambiguously, for the formulas $\varphi$ or $\neg \varphi$. All occurrences of $\pm_{i} \varphi$ within a single formula should be expanded in all possible ways to $\varphi$ and $\neg \varphi$ such that occurrences with the same index $i$ are expanded in the same way.

We are going to write formulas that induce a structure similar to that depicted in Fig. 1a, the bottom of which will represent the grid associated with (an instance of) a tiling problem. Fix $n>0$. We consider structures interpreting the proposition letters $u_{0}, \ldots, u_{n}, v_{0}, \ldots, v_{n}, p_{1}, \ldots, p_{n}$, $q_{1}, \ldots, q_{n}, z, o_{h}$ and $o_{v}$. Let $\Gamma_{1}$ be the set of all formulas:

$$
\begin{aligned}
& u_{0} \wedge v_{0} \wedge z \\
& \square\left(\neg\left(u_{i} \wedge u_{j}\right) \wedge \neg\left(v_{i} \wedge v_{j}\right)\right) \quad(0 \leq i<j \leq n) \\
& \bullet\left(u_{i} \wedge v_{j} \wedge z \rightarrow\right. \\
& \left.\left.\diamond\left(u_{i+1} \wedge v_{j} \wedge z \wedge \pm_{1} p_{i+1}\right)\right) \quad 0 \leq j \leq n\right) \\
& \sqcup\left(u_{i} \wedge v_{j} \wedge z \rightarrow\right. \\
& \left.\left.\diamond\left(u_{i} \wedge v_{j+1} \wedge z \wedge \pm_{1} q_{j+1}\right)\right) \quad 0 \leq j<n\right) \\
& \square\left(u_{i} \wedge \pm_{1} p_{k} \rightarrow \square\left(z \rightarrow \pm_{1} p_{k}\right)\right) \quad(1 \leq k \leq i \leq n) \\
& \square\left(v_{j} \wedge \pm_{1} q_{k} \rightarrow \square\left(z \rightarrow \pm_{1} q_{k}\right)\right) \quad(1 \leq k \leq j \leq n)
\end{aligned}
$$

Suppose $\mathfrak{A}$ is a transitive structure and $w_{0}$ a world of $\mathfrak{A}$ such that $\mathfrak{A} \models_{w_{0}} \Gamma_{1}$. We employ the following terminology. A world $w$ of $\mathfrak{A}$ has character $(i, j)$, for $i, j$ in the range $[0, n]$, if $\mathfrak{A} \models_{w} u_{i} \wedge v_{j}$. A z-world is a member of the smallest set $Z$ of worlds such that: $(i) w_{0} \in Z$; and (ii) if $w \in Z$, and $w^{\prime}$ is a direct successor of $w$ with $\mathfrak{A} \models{ }_{w^{\prime}} z$, then $w^{\prime} \in Z$. (Notice that the definition of z-world depends on $w_{0}$; where $w_{0}$ is not clear from context, we speak of a z-world relative to $w_{0}$.) Necessarily, every z-world is either identical to, or accessible from, $w_{0}$. For any z-world $w$, with character $(i, j)$, we define strings $s, t \in\{0,1\}^{*}$ of length $i$ and $j$, respectively, by setting $s[k]=1$ if and only if $\mathfrak{A}=_{w} p_{k}$ for all $k(1 \leq k \leq i)$, and $t[k]=1$ if and only if 


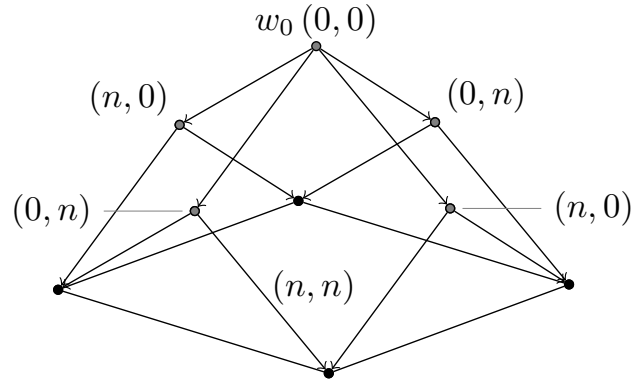

(a) The set of all z-worlds forming a (rather jumbled) 'ziggurat' under the direct successor relation. The world $w_{0}$, with character $(0,0)$, lies at the apex of the ziggurat, and the worlds with character $(n, n)$ form its base.

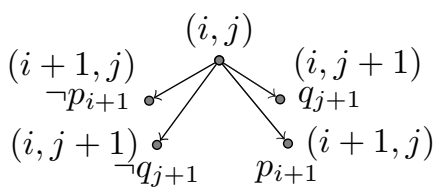

(b) The direct successors of a z-world with character $(i, j)$, where $0 \leq i<n$ and $0 \leq j<n$. Any such z-world has four direct successors: two with character $(i+1, j)$ and complementary values of $p_{i+1}$, and two with character $(i, j+1)$ and complementary values of $q_{j+1}$.

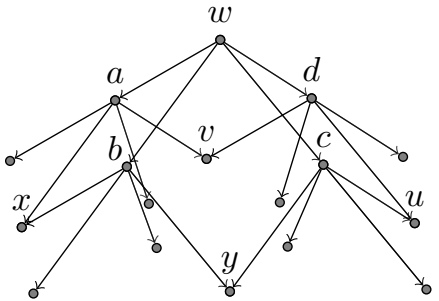

(c) Identifying $\mathrm{Z}$-worlds with the same indices using Formulas 11- 13. From every z-world $w$ with character $(i, j)$, we can access at most two z-worlds $a$ and $c$ with character $(i+1, j)$, at most two z-worlds $b$ and $d$ with character $(i, j+1)$, and at most four (not eight!) z-worlds $x, y, u$ and $v$ with character $(i+1, j+1)$

Figure 1: The set of z-worlds generated by Formulas (5)-13).

$\mathfrak{A} \models{ }_{w} q_{k}$ for all $k(1 \leq k \leq j)$. The quadruple $(i, j, s, t)$ is the index of $w$.

To see that Formulas (5)-(10) generate the structure in Fig. 1a note first that Formula (5) implies the existence of a $\mathrm{z}$-world $w_{0}$ with character $(0,0)$. Formulas (6) ensure that every $\mathrm{z}$-world has a unique character. If $0 \leq i<n$ and $0 \leq j<n$, then Formulas (7) and (8) imply that every zworld with character $(i, j)$ has four direct successors: two with character $(i+1, j)$ and complementary values of $p_{i+1}$, and two with character $(i, j+1)$ and complementary values of $q_{j+1}$ (Fig. 1b). Similarly, if $0 \leq i<n$ and $j=n$, or if $0 \leq j<n$ and $i=n$, every z-world with character $(i, j)$ has two direct successors.

Lemma 8. Suppose $\mathfrak{A} \models w_{0} \Gamma_{1}$. Let $w$ be a $z$-world with index $(i, j, s, t)$, and suppose $i^{\prime}, j^{\prime}, s^{\prime}, t^{\prime}$ satisfy: $(i) i \leq$ $i^{\prime} \leq n$; (ii) $j \leq j^{\prime} \leq n$; (iii) $i+j<i^{\prime}+j^{\prime}$; (iv) $s \preceq s^{\prime}$ and $\left\|s^{\prime}\right\|=i^{\prime}$; and $(v) t \preceq t^{\prime}$ and $\left\|t^{\prime}\right\|=j^{\prime}$. Then there exists $a$ $z$-world $w^{\prime}$, accessible from $w$, with index $\left(i^{\prime}, j^{\prime}, s^{\prime}, t^{\prime}\right)$.

Proof: Easy induction using Formulas (7)-10).

Lemma 9. Suppose $\mathfrak{A} \models{ }_{w_{0}} \Gamma_{1}$. For all $i(0 \leq i \leq n)$, all $j$ $(0 \leq j \leq n)$, all $s \in\{0,1\}^{*}(\|s\|=i)$ and all $t \in\{0,1\}^{*}$ $(\|t\|=j)$, there exists a $z$-world with index $(i, j, s, t)$.

Proof: From Lemma 8 and the fact that $w_{0}$ has index $(0,0, \epsilon, \epsilon)$

We now add formulas limiting the number of $\mathrm{z}$-worlds with any given character (see Fig. 1c). In particular, z-worlds will turn out to be uniquely identified by their indices. Let $\Gamma_{2}$ be the set of formulas:

$$
\begin{array}{rrr}
\nabla\left(u_{i} \wedge v_{j} \rightarrow\right. & (0 \leq i<n, \\
\left.\diamond_{\leq 1}\left(u_{i+1} \wedge v_{j} \wedge \pm_{1} p_{i+1}\right)\right) & 0 \leq j \leq n) \\
\sqcup\left(u_{i} \wedge v_{j} \rightarrow\right. & (0 \leq i \leq n, \\
\left.\diamond_{\leq 1}\left(u_{i} \wedge v_{j+1} \wedge \pm_{1} q_{j+1}\right)\right) & 0 \leq j<n)
\end{array}
$$

$$
\begin{array}{rlrl}
\nabla\left(u_{i} \wedge v_{j} \rightarrow\right. & & (0 \leq i<n, \\
\diamond_{\leq 1}\left(u_{i+1} \wedge v_{j+1} \wedge\right. & 0 & \leq j<n) \\
\left.\left. \pm_{1} p_{i+1} \wedge \pm_{2} q_{j+1}\right)\right) &
\end{array}
$$

Lemma 10. Suppose $\mathfrak{A} \models{ }_{w_{0}} \Gamma_{1} \cup \Gamma_{2}$. Then no two different $z$-worlds have the same index.

Proof: Order the pairs of integers in the range $[0, n]$ in some way such that $i+j<i^{\prime}+j^{\prime}$ implies $(i, j)<\left(i^{\prime}, j^{\prime}\right)$, and proceed by induction on the character $(i, j)$ of z-worlds, under this ordering.

Case 1: $w$ has character $(0,0)$. By definition, $w_{0}$ is the only z-world with character $(0,0)$, and hence the only z-world with index $(0,0, \epsilon, \epsilon)$.

Case 2: $w_{1}$ and $w_{2}$ have index $(i+1, j+1, s a, t b)$ where, $0 \leq i<n, 0 \leq j<n$ and $a, b \in\{0,1\}$. If $w_{1}$ and $w_{2}$ are $\mathrm{z}$-worlds, there exist $\mathrm{z}$-worlds $w_{1}^{\prime}$ and $w_{2}^{\prime}$ such that $w_{i}$ is a direct successor of $w_{i}^{\prime}(1 \leq i \leq 2)$. The possible characters of $w_{1}^{\prime}$ and $w_{2}^{\prime}$ are $(i+1, j)$ and $(i, j+1)$. If $w_{1}^{\prime}$ and $w_{2}^{\prime}$ have the same character, then they in fact have the same index (this follows from Formulas (9) and (10), and the fact that $w_{1}$ and $w_{2}$ have the same index). By inductive hypothesis, then, $w_{1}^{\prime}=w_{2}^{\prime}$. Hence, from Formulas 11 or (12), $w_{1}=w_{2}$ as required. If $w_{1}^{\prime}$ and $w_{2}^{\prime}$ have different characters, assume without loss of generality that $w_{1}^{\prime}$ has index $(i, j+1, s, t b)$, and $w_{2}^{\prime}$ has index $(i+1, j, s a, t)$. By Lemma 9, let $w^{*}$ be any z-world with index $(i, j, s, t)$. By Lemma 8, let $w_{1}^{\prime \prime}$ and $w_{2}^{\prime \prime}$ be z-worlds, accessible from $w^{*}$, with indices $(i, j+1, s, t b)$, and $(i+1, j, s a, t)$, respectively. By inductive hypothesis, $w_{1}^{\prime}=w_{1}^{\prime \prime}$, and $w_{2}^{\prime}=w_{2}^{\prime \prime}$ : that is to say, $w_{1}^{\prime}$ and $w_{2}^{\prime}$ are accessible from $w^{*}$. Therefore, so are $w_{1}$ and $w_{2}$. Formulas (13) then ensure that $w_{1}=w_{2}$.

Case 3: $w_{1}$ and $w_{2}$ have index $(i+1,0, s a, \epsilon)$ where $0 \leq$ $i<n$ and $a \in\{0,1\}$. The argument is similar to Case 2, and requires only Formulas (11).

Case 4: $w_{1}$ and $w_{2}$ have index $(0, j+1, \epsilon, t b)$ where $0 \leq$ $j<n$ and $b \in\{0,1\}$. The argument is similar to Case 2 , 


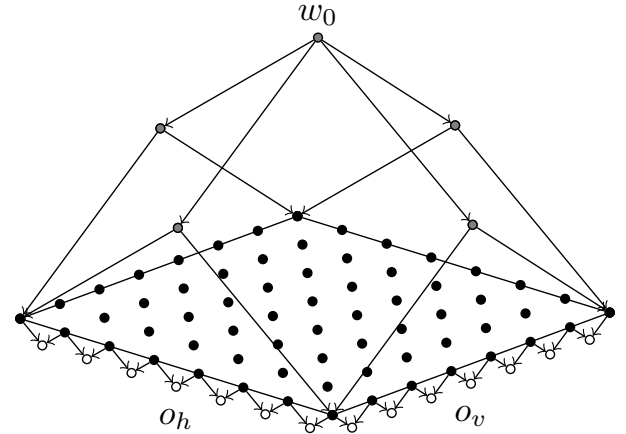

(a) The ziggurat, together with the grid at its base.

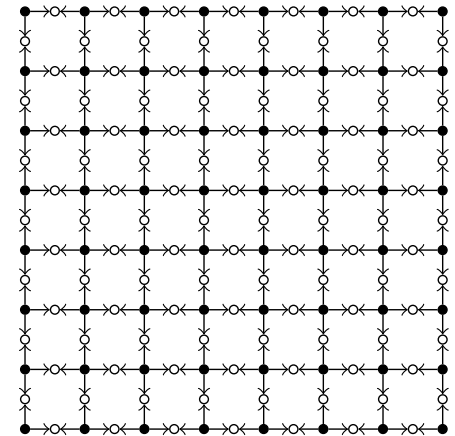

(b) The world arrangement for the grid

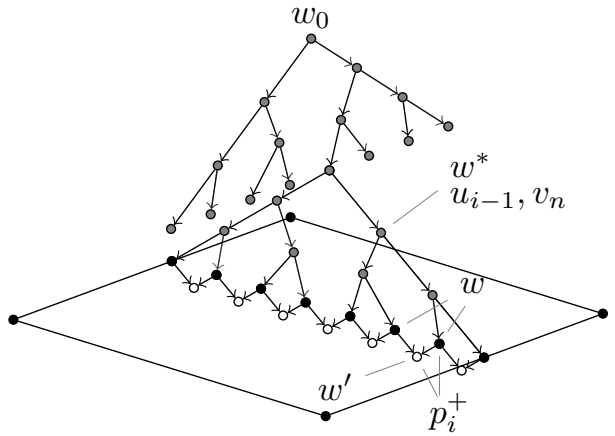

(c) An illustration of Formulas 17] and Lemma 14

Figure 2: Creating o-worlds (shown as a hollow dots) and the grid using Formulas (15)-20) ( $n=3$ ): g-worlds (shown as filled dots) are arranged according to their coordinates at the base; g-worlds which are horizontal neighbours in this grid have a common horizontal o-world successor, while g-worlds which are vertical neighbours in this grid have a common vertical o-world successor.

and requires only Formulas (12).

Lemma 11. Suppose $\mathfrak{A} \models{ }_{w_{0}} \Gamma_{1} \cup \Gamma_{2}$. Let $w_{1}, w_{2}$ be z-worlds with indices $\left(i_{1}, j_{1}, s_{1}, t_{1}\right)$ and $\left(i_{2}, j_{2}, s_{2}, t_{2}\right)$, respectively. Let $s^{*}$ be a common prefix of $s_{1}$ and $s_{2}$, and $t^{*}$ a common prefix of $t_{1}$ and $t_{2}$. Let $i^{*}=\left\|s^{*}\right\|$ and $j^{*}=\left\|t^{*}\right\|$. Then there exists a $z$-world $w^{*}$ with index $\left(i^{*}, j^{*}, s^{*}, t^{*}\right)$ such that each of $w_{1}$ and $w_{2}$ is either identical to, or accessible from, $w^{*}$.

Proof: By Lemma 9 there exists a $\mathrm{z}$-world $w^{*}$ with index $\left(i^{*}, j^{*}, s^{*}, t^{*}\right)$. If $i^{*}+j^{*}=i_{1}+j_{1}$ then $s^{*}=s_{1}$ and $t^{*}=t_{1}$, thus $w^{*}=w_{1}$ by Lemma 10 . Otherwise $i^{*}+j^{*}<$ $i_{1}+j_{1}$ and by Lemma 8 there exists a world $w_{1}^{\prime}$ accessible from $w^{*}$ with index $\left(i_{1}, j_{1}, s_{1}, t_{1}\right)$. By Lemma $10, w_{1}^{\prime}=w_{1}$. Thus $w_{1}$ is accessible from $w^{*}$. Similarly, one can show that either $w^{*}=w_{2}$ or $w_{2}$ is accessible from $w^{*}$.

The z-worlds of most interest are those with character $(n, n)$ - of which, by Lemmas 9 and 10 there are exactly $2^{2 n}$. We refer to such worlds as $g$-worlds (g for 'grid').

For any world $w$ (not just z-worlds), we define strings $s, t \in\{0,1\}^{*}$ of length $n$, by setting, for all $k(1 \leq k \leq n)$, $s[k]=1$ if and only if $\mathfrak{A} \models_{w} p_{k}$, and $t[k]=1$ if and only if $\mathfrak{A} \models{ }_{w} q_{k}$. We call the string $s$ the $x$-coordinate of $w$, and the string $t$ its $y$-coordinate. Notice that, if $w$ is a g-world, with index $(n, n, s, t)$, then its coordinates are $(s, t)$. The strings $s$ and $t$ may of course be regarded as integers in the range $\left[0,2^{n}-1\right]$, and in the sequel we equivocate freely between strings of length $n$ and the integers in this range they represent. The following abbreviations will be useful. If $1 \leq i \leq n$, we write $p_{i}^{*}$ for $\neg p_{i} \wedge p_{i+1} \wedge \cdots \wedge p_{n}$, and $p_{i}^{+}$ for $p_{i} \wedge \neg p_{i+1} \wedge \cdots \wedge \neg p_{n}$. Thus, $p_{i}^{*}$ and $p_{i}^{+}$characterize those worlds whose $x$-coordinates are of the forms

$$
a_{1} \cdots a_{i-1} 0 \overbrace{1 \cdots \cdots 1}^{n-i \text { times }} \quad a_{1} \cdots a_{i-1} 1 \overbrace{0 \cdots \cdots 0}^{n-i \text { times }}
$$

respectively. Observe that, if $s$ and $s^{\prime}$ are the respective strings (i.e. integers) depicted in (14), then $s^{\prime}=s+1$. The abbreviations $q_{i}^{*}$ and $q_{i}^{+}$will be used similarly.

We now write formulas which force the g-worlds to link up into a $2^{n} \times 2^{n}$ grid (see Fig. 2). This process is complicated by the fact that we are dealing with transitive accessibility relations. We employ proposition letters $o_{h}, o_{v}$, and refer to worlds satisfying these proposition letters as, respectively, horizontal o-worlds and vertical o-worlds ('o' stands for nothing in particular). The o-worlds' function is to glue the g-worlds into the desired grid pattern. Let $\Gamma_{3, h}$ be the set of formulas:

$$
\begin{array}{ll}
\square\left(u_{n} \wedge v_{n} \wedge p_{i}^{*} \rightarrow \diamond\left(o_{h} \wedge p_{i}^{+}\right)\right) & (1 \leq i \leq n) \\
\square\left(u_{n} \wedge v_{n} \wedge p_{i}^{+} \rightarrow \diamond\left(o_{h} \wedge p_{i}^{+}\right)\right) & (1 \leq i \leq n) \\
\square\left(u_{i-1} \wedge v_{n} \rightarrow \diamond_{\leq 1}\left(o_{h} \wedge p_{i}^{+}\right)\right) & (1 \leq i \leq n),
\end{array}
$$

and suppose $\mathfrak{A} \models{ }_{w_{0}} \Gamma_{1} \cup \Gamma_{2} \cup \Gamma_{3, h}$. Consider a g-world $w$ with coordinates $(s, t)$. If $0 \leq s<2^{n-1}$, then $w$ satisfies $p_{i}^{*}$ for some $i>0$, and so has a horizontal o-world successor by Formulas (15); likewise, if $0<s \leq 2^{n}-1$, then $w$ satisfies $p_{i}^{+}$for some $i>0$, and so has a horizontal o-world successor by Formulas (16). (Hence, if $0<s<2^{n-1}$, then $w$ has at least two horizontal o-world successors.) Finally, let $i$ be such that $1 \leq i \leq n$, and suppose that $w^{*}$ is a z-world with character $(i-1, n)$. Formulas (17) imply that there is at most one horizontal o-world accessible from $w^{*}$, and satisfying $p_{i}^{+}$(see Fig. 2c). The effect of these sets of formulas is illustrated in Fig. 2 and formalized in the following lemma:

Lemma 12. Suppose $\mathfrak{A} \models{ }_{w_{0}} \Gamma_{1} \cup \Gamma_{2} \cup \Gamma_{3, h}$. Let $w$ and $w^{\prime}$ be $g$-worlds with coordinates $(s, t)$ and $(s+1, t)$, respectively. Then there exists a horizontal o-world $u$ accessible from both $w$ and $w^{\prime}$ such that $\mathfrak{A} \models{ }_{u} p_{n}$ if and only if $\mathfrak{A} \models{ }_{w^{\prime}} p_{n}$.

Proof: Since $0 \leq s<s+1 \leq 2^{n}-1$, there exists $i$ such 
that $w$ satisfies $p_{i}^{*}$; thus $w^{\prime}$ satisfies $p_{i}^{+}$. From Formulas (15) and (16), there exist o-worlds $u, u^{\prime}$ both satisfying $p_{i}^{+}$, with $u$ accessible from $w$, and $u^{\prime}$ accessible from $w^{\prime}$. Clearly, $\mathfrak{A} \models_{u} p_{n}$ if and only if $\mathfrak{A} \models_{w^{\prime}} p_{n}$. By Lemma 11, there exists a $\mathrm{z}$-world $w^{*}$ with character $(i-1, n)$, for some $i$ $(1 \leq i \leq n)$, such that both $w$ and $w^{\prime}$, and hence both $u$ and $u^{\prime}$, are accessible from $w^{*}$. From Formulas (17), we have $u=u^{\prime}$.

Similarly, let $\Gamma_{3, v}$ be the set of formulas:

$$
\begin{array}{ll}
\square\left(u_{n} \wedge v_{n} \wedge q_{i}^{*} \rightarrow \diamond\left(o_{v} \wedge q_{i}^{+}\right)\right) & (1 \leq i \leq n) \\
\square\left(u_{n} \wedge v_{n} \wedge q_{i}^{+} \rightarrow \diamond\left(o_{v} \wedge q_{i}^{+}\right)\right) & (1 \leq i \leq n) \\
\square\left(u_{n} \wedge v_{i-1} \rightarrow \diamond_{\leq 1}\left(o_{v} \wedge q_{i}^{+}\right)\right) & (1 \leq i \leq n) .
\end{array}
$$

Lemma 13. Suppose $\mathfrak{A} \models_{w_{0}} \Gamma_{1} \cup \Gamma_{2} \cup \Gamma_{3, v}$. Let $w$ and $w^{\prime}$ be $g$-worlds with coordinates $(s, t)$ and $(s, t+1)$, respectively. Then there exists a vertical o-world $u$ accessible from both $w$ and $w^{\prime}$ such that $\mathfrak{A} \models_{u} q_{n}$ if and only if $\mathfrak{A} \models_{w^{\prime}} q_{n}$.

Proof: Analogous to Lemma 12.

Let $\Gamma=\Gamma_{1} \cup \Gamma_{2} \cup \Gamma_{3, h} \cup \Gamma_{3, v}$, and suppose $\mathfrak{A} \models_{w_{0}} \Gamma$. Lemmas 9 and 10 guarantee that, for all $s, t$ in the range $\left[0,2^{n}-1\right]$, there exists exactly one g-world with coordinates $(s, t)$; let $G$ be the set of all these $2^{2 n}$ g-worlds. And let $O_{v}$, $O_{h}$ be sets of horizontal and vertical o-worlds guaranteed by Lemmas 12 and 13 respectively. Thus, the frame of $\mathfrak{A}$ contains, as a subgraph, the configuration depicted in Fig. $2 \mathrm{~b}$ In short, the formulas $\Gamma$ manufacture a $2^{n} \times 2^{n}$ grid.

Conversely, it is easy to exhibit a model of $\Gamma$, using the diagrams of Fig. 2 as our guide, containing just such a grid.

Lemma 14. There exists a structure $\mathfrak{S}$ over a reflexive, transitive frame, and a world $w_{0}$ of $\mathfrak{S}$, such that $\mathfrak{S} \models w_{0} \Gamma$.

Proof: For $h$ and $v$ distinct symbols, define the sets:

$$
\begin{aligned}
Z= & \{(i, j, s, t) \mid 0 \leq i \leq n ; 0 \leq j \leq n ; \\
\left.s, t \in\{0,1\}^{*} ;\|s\|=i \text { and }\|t\|=j\right\} & \\
G= & \left\{(n, n, s, t) \mid s, t \in\{0,1\}^{*} \text { and }\|s\|=\|t\|=n\right\} \\
O_{h}= & \left\{(h, s, t) \mid s, t \in\{0,1\}^{*} ; s \notin\{0\}^{*} ;\|s\|=\|t\|=n\right\} \\
O_{v}= & \left\{(v, s, t) \mid s, t \in\{0,1\}^{*} ; t \notin\{0\}^{*} ;\|s\|=\|t\|=n\right\} .
\end{aligned}
$$

Note that $G \subseteq Z$. Define the binary relations $R_{Z} \subseteq Z \times Z$, $R_{h} \subseteq G \times O_{h}$ and $R_{v} \subseteq G \times O_{v}$ by:

$$
\begin{aligned}
& R_{Z}=\left\{\left\langle(i, j, s, t),\left(i^{\prime}, j^{\prime}, s^{\prime}, t^{\prime}\right)\right\rangle\right. \\
& R_{h}=\left\{\left\langle(n, n, s, t),\left(h, s^{\prime}, t^{\prime}\right)\right\rangle\right. \\
&\left.\mid t^{\prime}=t ; s \leq s^{\prime} \leq n \text { and } 1 \leq s^{\prime} \leq s+1\right\} \\
& R_{v}=\left\{\left\langle(n, n, s, t),\left(v, s^{\prime}, t^{\prime}\right)\right\rangle\right. \\
&\left.\quad \mid s^{\prime}=s ; t \leq t^{\prime} \leq n \text { and } 1 \leq t^{\prime} \leq t+1\right\} .
\end{aligned}
$$

Finally, let $S=Z \cup O_{h} \cup O_{v}$, and let $R_{S}$ be the reflexive, transitive closure of $R_{Z} \cup R_{h} \cup R_{v}$. Thus, $\left(S, R_{S}\right)$ is a reflexive, transitive frame. Define a valuation $V$ on $\left(S, R_{S}\right)$ by interpreting the proposition letters as follows:

$$
z^{\mathfrak{S}}=Z ; \quad o_{h}^{\mathfrak{S}}=O_{h} ; \quad o_{v}^{\mathfrak{S}}=O_{v}
$$

$$
\begin{aligned}
u_{i}^{\mathfrak{S}}= & \left\{(i, j, s, t) \in Z \mid 0 \leq j \leq n ; s, t \in\{0,1\}^{*}\right\} \\
v_{j}^{\mathfrak{S}}= & \left\{(i, j, s, t) \in Z \mid 0 \leq i \leq n ; s, t \in\{0,1\}^{*}\right\} \\
p_{i}^{\mathfrak{S}}= & \left\{\left(i^{\prime}, j, s, t\right) \in Z \mid i^{\prime} \geq i, s[i]=1\right\} \cup \\
& \left\{(h, s, t) \in O_{h} \mid s[i]=1\right\} \cup \\
& \left\{(v, s, t) \in O_{v} \mid s[i]=1\right\} \\
q_{j}^{\mathfrak{S}}= & \left\{\left(i, j^{\prime}, s, t\right) \in Z \mid j^{\prime} \geq j, t[j]=1\right\} \cup \\
& \left\{(h, s, t) \in O_{h} \mid t[j]=1\right\} \cup \\
& \left\{(v, s, t) \in O_{v} \mid t[j]=1\right\} .
\end{aligned}
$$

Denote by $\mathfrak{S}$ the structure $\left(S, R_{S}, V\right)$. Let $w_{0} \in Z$ be the element $(0,0, \epsilon, \epsilon)$. Thus, $\mathfrak{S} \models{ }_{w_{0}} \Gamma_{1}$, and, relative to $w_{0}$, the Z-worlds of $\mathfrak{S}$ are simply the elements of $Z$. It is obvious that, for every $w=(i, j, s, t) \in Z$, the index of $w$ is $w$ itself; moreover, for every $w=(h, s, t) \in o_{h}$ and every $w=(v, s, t) \in o_{v}$, the coordinates of $w$ are $(s, t)$.

We now show that $\mathfrak{S} \models_{w_{0}} \Gamma$. The truth at $w_{0}$ of Formulas (5)-(20) except for Formulas (17) and (20) is immediate. To demonstrate the truth of Formulas (17), let $1 \leq i \leq n$, and fix any world $w^{*}$ of $\mathfrak{S}$ such that $\mathfrak{S} \models_{w^{*}} u_{i-1} \wedge v_{n}$ (see Fig. 2c). We may write $w^{*}=\left(i-1, n, s^{*}, t^{*}\right)$, where $\left\|s^{*}\right\|=i-1$ and $\left\|t^{*}\right\|=n$. Now suppose $w^{\prime}$ is any world of $\mathfrak{S}$ such that $\left\langle w^{*}, w^{\prime}\right\rangle \in R_{S}$ and $\mathfrak{S} \models{ }_{w^{\prime}} o_{h} \wedge p_{i}^{+}$. Again, we may write $w^{\prime}=\left(h, s^{\prime}, t^{\prime}\right)$, where $s^{\prime}$ and $t^{\prime}$ are bit-strings of length $n$. We claim that $s^{\prime}=s^{*} 10 \ldots 0$ and $t^{\prime}=t^{*}$. But there is at most one world in $\mathfrak{S}$ satisfying $o_{h}$ and having coordinates $\left(s^{*} 10 \ldots 0, t^{*}\right)$; hence, $\mathfrak{S} \models_{w_{0}} \square\left(u_{i-1} \wedge v_{n} \rightarrow\right.$ $\left.\diamond_{\leq 1}\left(o_{h} \wedge p_{i}^{+}\right)\right)$, as required.

To prove the claim, observe that, by construction of $\mathfrak{S}$, there exists $w \in G$ such that $\left\langle w^{*}, w\right\rangle \in R_{S}$ and $\left\langle w, w^{\prime}\right\rangle \in$ $R_{S}$. Pick any such $w$ and let it have coordinates $(s, t)$. By the definition of $R_{S}$ (and the fact that $\left\|t^{*}\right\|=n$ ), we have: (i) $t^{*}=t=t^{\prime}$, (ii) $s^{*} \preceq s$, and (iii) $s^{\prime}=s$ or $s^{\prime}=s+1$. Referring to Fig. 2C, the worlds $w^{*}, w$ and $w^{\prime}$ can be reached from $w_{0}$ by traversing two trees of z-worlds: an upper tree, whose leaves have characters $(0, n)$, and a lower tree, whose elements have characters $(i, n)(0 \leq i \leq n)$. The world $w^{*}$ in the lower tree, has character $(i-1, n) ; w^{\prime}$ is a horizontal o-world reachable from $w^{*} ; w$ is its predecessor g-world. Now, since $\mathfrak{S} \models_{w^{\prime}} o_{h} \wedge p_{i}^{+}$, we have $s^{\prime}=s^{\prime \prime} 10 \ldots 0$ for some string $s^{\prime \prime}$ with $\left\|s^{\prime \prime}\right\|=i-1$. Since $s$ is either $s^{\prime}$ or $s^{\prime}-1$, we have either $s=s^{\prime \prime} 10 \ldots 0$ or $s=s^{\prime \prime} 01 \ldots 1$. Since $s^{*} \preceq s$ and $\left\|s^{*}\right\|=i-1$, we have $s^{\prime \prime}=s^{*}$. Thus, $s^{\prime}=s^{*} 10 \ldots 0$ and $t^{\prime}=t^{*}$, proving the claim.

The case of Formulas (20) is treated analogously.

Now we are in a position to encode any exponential tiling problem, $(C, H, V, p)$ in our logic. We regard colours $c \in C$ as (fresh) proposition letters. Suppose $\mathfrak{A}$ is transitive and $\mathfrak{A} \models w_{0} \Gamma$, and let $\mathfrak{A}$ additionally interpret the proposition letters $c \in C$. By Lemmas $9,10,12$ and 13 , the frame of $\mathfrak{A}$ contains the arrangement of Fig. $2 \mathrm{~b}$ as a subgraph, which we may partition into the sets $G$ (the g-worlds), $O_{h}$ (the horizontal o-worlds) and $O_{v}$ (the vertical o-worlds). 
Intuitively, for any world $w \in G, c$ represents the colour of $w$ in some (putative) tiling of $G$. Now we write formulas to ensure that the colours form a tiling for $(C, H, V, p)$. Define $\Delta$ to be the following set of formulas:

$$
\begin{aligned}
& \square\left(u_{n} \wedge v_{n} \rightarrow(\bigvee C \wedge \bigwedge\{\neg c \vee \neg d \mid c \neq d\})\right) \\
& \begin{array}{c}
\square\left(u_{n} \wedge v_{n} \wedge \pm_{1} p_{n} \wedge c \rightarrow\right. \\
\left.\square\left(o_{h} \wedge \pm_{1} p_{n} \rightarrow c\right)\right)
\end{array} \quad(c \in C) \\
& \square\left(u_{n} \wedge v_{n} \wedge \pm_{1} p_{n} \wedge c \rightarrow\right. \\
& \left.\square\left(o_{h} \wedge \neg\left( \pm_{1} p_{n}\right) \rightarrow \neg d\right)\right) \\
& (\langle c, d\rangle \notin H) \\
& \square\left(u_{n} \wedge v_{n} \wedge \pm_{1} q_{n} \wedge c \rightarrow\right. \\
& \left.\square\left(o_{v} \wedge \pm_{1} q_{n} \rightarrow c\right)\right) \\
& (c \in C) \\
& \square\left(u_{n} \wedge v_{n} \wedge \pm_{1} q_{n} \wedge c \rightarrow\right. \\
& \left.\square\left(o_{v} \wedge \neg\left( \pm_{1} q_{n}\right) \rightarrow \neg d\right)\right) \\
& (\langle c, d\rangle \notin V)
\end{aligned}
$$

Formula (21) ensures that every g-world is assigned a unique colour. Using Lemma 12. Formulas (22) ensure every horizontal o-world has the same colour as the g-world 'immediately to the right'. Together with Formulas (21) and (23), this ensures that the g-worlds satisfy the horizontal tiling constraints. Likewise, Formulas (21), (24), and (25) ensure that the g-worlds satisfy the vertical tiling constraints.

Lemma 15. Suppose $\mathfrak{A}$ is transitive, and $\mathfrak{A} \models w_{0} \Gamma \cup \Delta$. For all $s, t$ in the range $\left[0,2^{n}-1\right]$, define $f(s, t)=c$ if $\mathfrak{A} \models{ }_{w} c$ for some $g$-world $w$ with coordinates $(s, t)$. Then $f$ is well-defined, and is in fact a tiling for $(C, H, V)$.

Proof: Immediate.

Now suppose $\bar{d}=d_{0}, \ldots, d_{m-1}$ is an $m$-tuple of elements of $C$. Let $\pi_{0}$ be the formula:

$$
\square\left(z \wedge \neg p_{1} \wedge \cdots \wedge \neg p_{n} \wedge \neg q_{1} \wedge \cdots \wedge \neg q_{n} \rightarrow d_{0}\right)
$$

implying that any g-world with coordinates $(0,0)$ has colour $d_{0}$; and let the formulas $\pi_{1}, \ldots, \pi_{m-1}$ be defined analogously, assigning colours $d_{1}, \ldots, d_{m-1}$ to the g-worlds with coordinates $(1,0), \ldots,(m-1,0)$. Denote by $\Theta_{\bar{d}}$ the set of all these formulas.

Lemma 16. Suppose $\mathfrak{A}$ is transitive, with $\mathfrak{A} \models{ }_{w_{0}} \Gamma \cup \Delta \cup \Theta_{\bar{d}}$, and let the tiling $f$ be as defined in Lemma 15 Then $\bar{d}$ is an initial configuration for $f$.

Proof: Immediate.

Thus, we have:

Lemma 17. Let $\mathcal{K}$ be any class of frames satisfying $\operatorname{Tr} \supseteq \mathcal{K} \supseteq \operatorname{Tr} \cap$ Rfl. The problem $\mathcal{G} \mathcal{M}_{\mathcal{K}}$-Sat is NExpTimehard. It remains NExpTime-hard, even when all numerical subscripts in modal operators are bounded by 1.

Proof: We reduce any exponential tiling problem $(C, H, V, p)$ to the problem $\mathcal{G} \mathcal{M}_{\mathcal{K}}$-Sat. Fix $(C, H, V, p)$, and let an instance $\bar{d}$ of size $m$ be given. Write $n=p(m)$. Consider the conjunction $\varphi_{\bar{d}}$ of all formulas in the set
$\Gamma \cup \Delta \cup \Theta_{\bar{d}}$. We claim that the following are equivalent: (i) $\varphi_{\bar{d}}$ is satisfiable over $\operatorname{Tr} \cap \mathrm{Rfl}$; (ii) $\varphi_{\bar{d}}$ is satisfiable over $\operatorname{Tr}$; (iii) $\bar{d}$ is a positive instance of $(C, H, V, p)$. The implication $(i) \Rightarrow$ (ii) is trivial. For $(i i) \Rightarrow(i i i)$, suppose $\mathfrak{A} \models w_{0} \Gamma \cup \Delta \cup \Theta_{\bar{d}}$, with $\mathfrak{A}$ transitive. Lemmas 15 and 16 then guarantee the existence of a tiling $f$ of size $2^{n}$ for $(C, H, V)$, with initial configuration $\bar{d}$. For $(i i i) \Rightarrow(i)$, suppose $f$ is a tiling for $(C, H, V)$ of size $2^{n}$, with initial configuration $\bar{d}$. Taking $\mathfrak{S}$ and $w_{0}$ to be as in the proof of Lemma 14, we expand $\mathfrak{S}$ to a structure $\mathfrak{S}^{*}$ by setting $c^{\mathfrak{S}^{*}}=\{(n, n, s, t),(h, s, t),(v, s, t) \mid f(s, t)=c\}$ for every proposition letter $c \in C$. It is obvious that $\mathfrak{S}^{*} \models{ }_{w_{0}} \Delta \cup \Theta_{\bar{d}}$.

Theorem 5 follows from Corollary 11 and Lemma 17 noting that $\operatorname{Rfl} \cap \operatorname{Tr}=\operatorname{Rfl} \cap \operatorname{Ser} \cap \operatorname{Tr} \subseteq \operatorname{Ser} \cap \operatorname{Tr} \subseteq \operatorname{Tr}$.

\section{CONCLUSION}

In this paper, we have investigated the computational complexity of $\mathcal{G} \mathcal{M}_{\cap \mathcal{F}}$-Sat, the satisfiability problem for graded modal logic over any frame class $\bigcap \mathcal{F}$, where $\mathcal{F} \subseteq\{$ Rfl, Ser, Sym, Tr, Eucl $\}$. The results are as follows. Suppose first that Eucl $\notin \mathcal{F}$ and $\operatorname{Tr} \notin \mathcal{F}$. Then Theorem 3 states that $\mathcal{G} \mathcal{M}_{\cap \mathcal{F}}$-Sat is PSpace-complete. Suppose next that Eucl $\in \mathcal{F}$ or $\{\mathrm{Sym}, \mathrm{Tr}\} \subseteq \mathcal{F}$. Then Theorem 4 states that $\mathcal{G} \mathcal{M}_{\cap \mathcal{F}}$-Sat is NP-complete. Suppose finally that Eucl, Sym $\notin \mathcal{F}$, but $\operatorname{Tr} \in \mathcal{F}$. Then Theorem [5] states that $\mathcal{G} \mathcal{M}_{\cap \mathcal{F}}$-Sat is NExpTime-complete. All these results hold under both unary and binary coding of numerical subscripts.

\section{ACKNOWLEDGMENT}

The authors would like to thank Prof. Ulrike Sattler for helpful discussions. Yevgeny Kazakov is supported by the EPSRC, grant number EP/G02085X. Ian Pratt-Hartmann is supported by the EPSRC, grant number EP/F069154.

\section{REFERENCES}

[1] P. Blackburn, M. de Rijke, and Y. Venema, Modal Logic, ser. Cambridge Tracts in Theoretical Computer Science. Cambridge: Cambridge University Press, 2001.

[2] R. Ladner, "The computational complexity of provability in systems of modal propositional logic," SIAM J. on Comp., vol. 6, pp. 467-480, 1977.

[3] M. Vardi, "Why is modal logic so robustly decidable?" in Descriptive Complexity and Finite Models, ser. DIMACS, vol. 31. AMS, 1997, pp. 149-184.

[4] S. Tobies, "PSPACE reasoning for graded modal logics," $J$. of Logic and Computation, vol. 11, no. 1, pp. 85-106, 2001.

[5] B. Hollunder and F. Baader, "Qualifying number restrictions in concept languages," in Proc. of KR 1991. Morgan Kaufmann, 1991, pp. 335-346.

[6] K. Fine, "In so many possible worlds," Notre Dame Journal of Formal Logic, vol. XIII, no. 4, pp. 516-520, 1972. 
[7] C. Cerrato, "Decidability by filtration for graded normal logics (Graded modalities V)," Studia Logica, vol. 53, pp. 61-74, 1994.

[8] Y. Kazakov, U. Sattler, and E. Zolin, "How many legs do I have? Non-simple roles in number restrictions revisited," in Proc. of LPAR 2007, ser. LNCS, vol. 4790. Springer, 2007.

[9] W. van der Hoek and M. de Rijke, "Counting objects," $J$. Logic and Computation, vol. 5, no. 3, pp. 325-345, 1995.

[10] M. Fattorosi-Barnaba and F. de Caro, "Graded modalities I," Studia Logica, vol. 44, pp. 197-221, 1985.

[11] F. de Caro, "Graded modalities II," Studia Logica, vol. 47, pp. 1-10, 1988.

[12] M. Fattorosi-Barnaba and C. Cerrato, "Graded modalities III (the completeness and compactness of $\mathrm{S}^{0}$ )," Studia Logica, vol. 47, pp. 99-110, 1988.

[13] C. Cerrato, "General canonical models for graded normal logics (Graded modalities IV)," Studia Logica, vol. 49, pp. 241-252, 1990.

[14] F. Baader, D. Calvanese, D. L. McGuinness, D. Nardi, and P. F. Patel-Schneider, Eds., The Description Logic Handbook. Cambridge University Press, 2007.

[15] I. Horrocks, U. Sattler, and S. Tobies, "Practical reasoning for very expressive description logics," Logic Journal of the $I G P L$, vol. 8, no. 3, 2000.

[16] Y. Kazakov and I. Pratt-Hartmann, "A note on the complexity of the satisfiability problem for graded modal logics," in Proc. of LICS 2009. IEEE Computer Society, 2009, in press.

[17] V. Kuncak and M. Rinard, "Towards efficient satisfiability checking for boolean algebra with Presburger arithmetic," in Proc. of CADE 2007, ser. LNCS, vol. 4603. Springer, 2007, pp. 215-230.

[18] I. Pratt-Hartmann, "On the computational complexity of the numerically definite syllogistic and related logics," Bulletin of Symbolic Logic, vol. 14, no. 1, pp. 1-28, 2008.

[19] E. Börger, E. Grädel, and Y. Gurevich, The Classical Decision Problem. Springer, 1997. 